\title{
Pint lincRNA connects the p53 pathway with epigenetic silencing by the Polycomb repressive complex 2
}

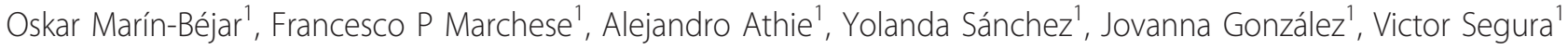 \\ , Lulu Huang ${ }^{2}$, Isabel Moreno ${ }^{3}$, Alfons Navarro ${ }^{4}$, Mariano Monzó ${ }^{4}$, Jesús García-Foncillas ${ }^{5}$, John L Rinn 6 , \\ Shuling Guo ${ }^{2}$ and Maite Huarte ${ }^{1 *}$
}

\begin{abstract}
Background: The p53 transcription factor is located at the core of a complex wiring of signaling pathways that are critical for the preservation of cellular homeostasis. Only recently it has become clear that p53 regulates the expression of several long intergenic noncoding RNAs (lincRNAs). However, relatively little is known about the role that lincRNAs play in this pathway.

Results: Here we characterize a lincRNA named Pint (p53 induced noncoding transcript). We show that Pint is a ubiquitously expressed lincRNA that is finely regulated by p53. In mouse cells, Pint promotes cell proliferation and survival by regulating the expression of genes of the TGF- $\beta$, MAPK and p53 pathways. Pint is a nuclear lincRNA that directly interacts with the Polycomb repressive complex 2 (PRC2), and is required for PRC2 targeting of specific genes for H3K27 tri-methylation and repression. Furthermore, Pint functional activity is highly dependent on PRC2 expression. We have also identified Pint human ortholog (PINT), which presents suggestive analogies with the murine lincRNA. PINT is similarly regulated by $\mathrm{p} 53$, and its expression significantly correlates with the same cellular pathways as the mouse ortholog, including the p53 pathway. Interestingly, PINT is downregulated in colon primary tumors, while its overexpression inhibits the proliferation of tumor cells, suggesting a possible role as tumor suppressor.
\end{abstract}

Conclusions: Our results reveal a p53 autoregulatory negative mechanism where a lincRNA connects p53 activation with epigenetic silencing by PRC2. Additionally, we show analogies and differences between the murine and human orthologs, identifying a novel tumor suppressor candidate lincRNA.

Keywords: lincRNA, non-coding RNA, p53, gene regulation, Polycomb repressive complex 2

\section{Background}

How cells coordinate and integrate information to produce adequate gene-expression output is still an unsolved question with important implications for biology and health. Even the slightest perturbation of cellular networks can affect homeostasis and lead to cell transformation. Of these cellular networks, the p53 pathway is possibly the most relevant for preservation of cellular homeostasis. The transcription factor p53 is located at the core of a complex

\footnotetext{
* Correspondence: maitehuarte@unav.es

${ }^{1}$ Center for Applied Medical Research, University of Navarra, 55 Pio XII

Avenue., 31008 Pamplona, Spain

Full list of author information is available at the end of the article
}

wiring of signaling pathways, and it has been proposed as the master regulator of cell fate. The importance of the tumor suppressing functions of p53 is shown by its high mutation frequency in cancers and by the highly tumorigenic phenotype of p53 null mice [1].

We and others have shown that long intergenic non-coding RNAs (lincRNAs) are part of the p53 transcriptional network [2-4]. LincRNAs are intergenic transcripts longer than 200 nucleotides that lack functional open reading frames (ORFs). Although thousands of lincRNAs exist, only a relatively small number have been studied in some depth, indicating that lincRNAs have roles in numerous physiological processes that involve gene regulation $[5,6]$.
Ciomed Central

(c) 2013 Marín-Béjar et al.; licensee BioMed Central Ltd. This is an open access article distributed under the terms of the Creative Commons Attribution License (http://creativecommons.org/licenses/by/2.0), which permits unrestricted use, distribution, and reproduction in any medium, provided the original work is properly cited. 
Many of these lincRNAs have been shown to act as molecular scaffolds that hold and guide chromatin complexes [7-9]. In particular, several lincRNAs have been found to be associated with the Polycomb repressive complex 2 (PRC2) in a number of biological contexts, modulating PRC2-specific targeting of genes [8,10,11]. PRC2 is composed of three core components: Suppressor of zeste 12 (Suz12), Embryonic Ectoderm Development (EED), and the H3K27 histone methyl transferase Enhancer of zeste homolog 2 (Ezh2). PRC2 represses gene expression by catalyzing H3K27 tri-methylation and modulating chromatin structure [12], and is closely linked with the aberrant proliferation of cancer cells. For instance, the Suz12 subunit is overexpressed in colon and breast cancers [13], and Ezh2 is upregulated in a number of tumors, including Hodgkin lymphoma, prostate cancer, and breast cancer $[14,15]$. Moreover, Ezh2 expression is associated with poor prognosis, and is an indication of the metastatic potential of a tumor $[15,16]$. Similarly, alterations in expression of lincRNAs in cancer have been reported, implicating lincRNAs as possible attractive therapeutic targets $[17,18]$.

In a previous work. we used mouse cell lines combined with custom microarrays to monitor the differential expression of lincRNAs, and found that p53 specifically activated several lincRNAs. We characterized one of them, lincRNA-p21, which was found to function as a transcriptional repressor [3]. However, the contribution of lincRNAs to p53 biology and to cancer still remains largely unexplored.

Here, we expand this knowledge by characterizing Pint. We show that Pint is a ubiquitously expressed mouse lincRNA that is a direct p53 transcriptional target. Pint acts as a positive regulator of cell proliferation and survival, affecting the expression of hundreds of genes, including a fraction of the p53 transcriptional network. PINT interacts with PRC2 and is required for PRC2 targeting of specific genes for $\mathrm{H} 3 \mathrm{~K} 27$ tri-methylation and repression. We also show that the PINT human ortholog is similarly regulated by $\mathrm{p} 53$. Interestingly, whereas in normal tissue, PINT shows a significant inverse correlation with the p53 pathway, it is downregulated in colorectal cancer, and its enforced expression inhibits the proliferation of tumor cells. To our knowledge, the results presented here represent the first experimentally supported connection between the p53 pathway and Polycomb epigenetic regulation mediated by a lincRNA. Moreover, the data suggest that PINT may serve as a novel tumor suppressor.

\section{Results}

\section{Pint, a long non-coding RNA transcriptionally regulated by $\mathrm{p} 53$}

Despite p53 being one of the most studied biological molecules, it has only recently become clear that p53 directly regulates numerous small and large non-coding RNAs [2-4]. In addition, the nature of these transcripts and the role that they play in this tumor suppressor pathway remains relatively unexplored. By using custom tiling microarrays, we previously identified multiple polyadenylated non-coding transcripts that were induced upon expression of p53 in mouse model systems [3]. In that study, we showed that one of the most significantly induced non-coding RNAs, previously named lincRNAMkln1 (which from this point we refer to as Pint (p53induced non-coding transcript)), is generated from an intergenic region located on chromosome 6 (Figure 1A; see Additional file 1: Figure S1A). To investigate the regulation of this genomic region by $\mathrm{p} 53$, we searched for $\mathrm{p} 53$ binding motifs using a method that scores genetic conservation based on the evolutionary substitution pattern inferred for the binding site locus [19]. We found three putative p53 response elements (p53RE-1, p53RE-2, and p53RE-3) inside this region with a high Pi LOD score (>110) (Figure 1A; see Additional file 2).

To experimentally test the biological activity of these regulatory elements, we first cloned the genomic regions of p53RE-1, p53RE-2, and p53RE-3 into a reporter vector, and transfected them into p53-reconstituted $\left(\mathrm{p} 53^{+/+}\right)$or non-reconstituted ( $\left.553^{-/-}\right) \mathrm{p} 53^{\mathrm{LSL} / \mathrm{LSL}}$ mouse embryonic fibroblasts (MEFs) to test the reporter-gene induction in the presence or absence of p53. The tested sequences were able to drive transcription of the reporter gene in the presence but not in the absence of p53, with the transcriptional induction being even higher when the $\mathrm{p} 53^{+/+}$cells were treated with the DNA-damaging drug doxorubicin (Figure 1B).

Next, to verify the activity of the p53REs in Pint locus, we performed p53 chromatin immunoprecipitation (ChIP), which showed specific and robust binding of p53 to all three predicted p53REs in the endogenous locus upon doxorubicin-induced DNA damage in $\mathrm{p} 53^{+/+}$, but not $\mathrm{p} 53^{-/-}$cells (Figure $1 \mathrm{C}$ ).

To further confirm our observations, we analyzed previously published p53 ChIP sequencing (ChIP-seq) data from mouse embryonic stem cells (mESCs) (total and phosphorylated p53) [20] and MEFs (total p53) [21]. In mESCs, we identified ChIP-seq peaks of total and phosphorylated p53 after doxorubicin treatment at positions corresponding to Pint p53RE-1 and p53RE-2, but not at the position corresponding to p53RE-3 (see Additional file 1, Figure S1), suggesting that p53RE-3 activity may be cell type-dependent. The previously published p53 ChIP-seq data from MEFs showed specific peaks at the Pint p53RE-1, p53RE-2, and p53RE-3 locations in doxorubicin p53 wildtype but not p53-null MEFs, in agreement with our results (Figure 1D). Together, these data confirm that the Pint genomic locus is controlled by $\mathrm{p} 53$, which directly binds to the harbored regulatory sequences. 
A

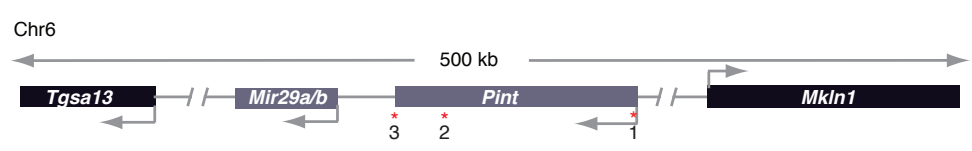

B

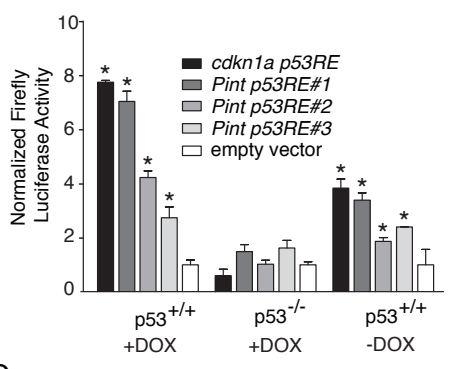

D

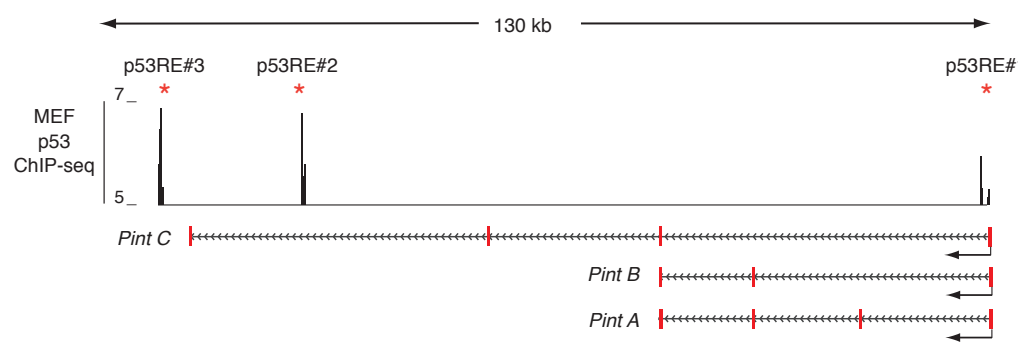

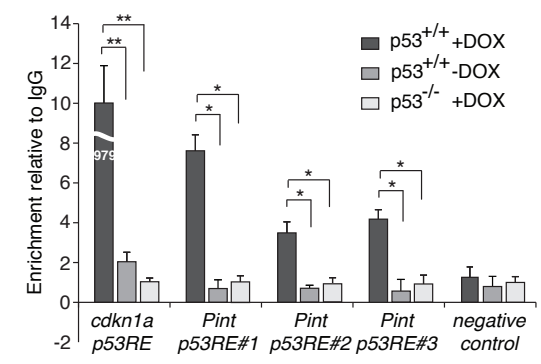

$\square \mathrm{p} 53^{+/+}+\mathrm{DOX}$ $\square \mathrm{p} 53^{+/+}-\mathrm{DOX}$

C
$E$

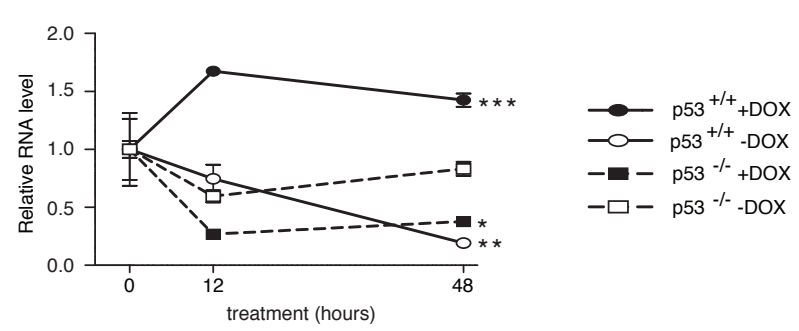

F

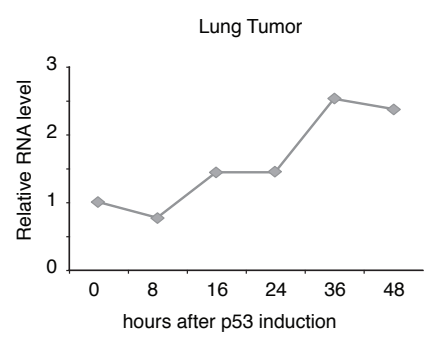

G

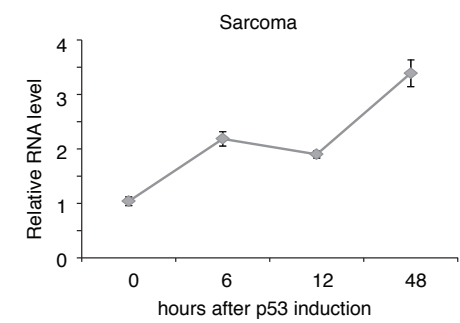

Figure 1 Pint is a p53-regulated long intergenic non-coding RNA (lincRNA). (A) Schematic representation of the Pint genomic locus. Asterisks represent p53 response elements (p53REs). (B) Relative firefly luciferase expression driven by genomic sequences containing p53REs in p53-restored $\mathrm{p} 53^{\mathrm{LSL} / \mathrm{LSL}}\left(\mathrm{p5} 3^{+/+}\right)$or $\mathrm{p} 53^{\mathrm{LSL} / \mathrm{LSL}}\left(\mathrm{p} 53^{-/}\right)$cells treated with doxorubicin. Values were normalized by Renilla levels and are the mean \pm standard deviation (SD) of three biological replicates. Asterisks represent significant differences determined by $t$-test relative to the same plasmid transfected in doxorubicin (DOX)-treated p53\%. (C) Effect on Pint p53RE-1, p53RE-2, and p53RE-3, Cdkn1a p53RE, or an irrelevant region (control) of p53 chromatin immunoprecipitation (ChIP) enrichment in p53-restored $\mathrm{p} 53^{\mathrm{LSL} / \mathrm{LSL}}\left(\mathrm{p} 53^{+/+}\right)$or $\mathrm{p} 53^{\mathrm{LSL} / \mathrm{SL}}\left(\mathrm{p} 53^{-/}\right)$cells treated with doxorubicin (+DOX) or left untreated $(-D O X)$. Enrichment values are relative to input and the mean \pm SD of three biological replicates. Asterisks represent statistical significant differences from the control as determined by $t$-test $\left({ }^{*} P<0.05\right.$, $\left.{ }^{* *} P<0.01\right)$. (D) (Top) p53 ChIP sequencing (ChIP-seq) peaks from mouse embryonic fibroblasts (MEFs) treated with doxorubicin [21]. Positions of p53REs are indicated by red asterisks. (Bottom) Pint variants identified by $5^{\prime}$ and $3^{\prime}$ rapid amplification of

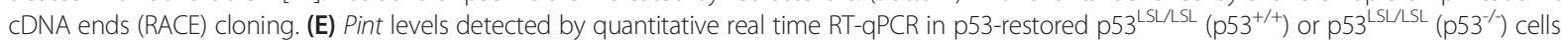
treated with $150 \mathrm{nM}$ doxorubicin (+DOX) or left untreated (-DOX) for the indicated time (values represent the mean \pm SD of three biological replicates, and asterisks represent significant differences of Pint level at 48 hours relative to the doxorubicin-treated $\mathrm{p} 53^{--}$cells). (F,G) Pint levels at different times after p53 restoration in (F) lung tumor $\mathbf{( G )}$ and sarcoma cell lines. Values are the mean \pm SD of four replicates. 
To better define the length and structure of the transcripts produced in this p53-regulated locus, we carried out 5' and 3' rapid amplification of cDNA ends (RACE) cloning from doxorubicin-treated MEFs. We detected a transcript of 1157 nucleotides and 4 exons, similar to the annotated EST BC145649, isoform that we named Pint A. Additionally, we cloned two shorter transcripts of 516 (Pint B) and 659 (Pint C) nucleotides, which share three and two exons, respectively, with the longer Pint $A$ variant (Figure 1D; see Additional file 1: Figure S2A).

To obtain additional information on Pint transcript structure, we analyzed publicly available RNA-seq data from mouse heart, thymus, and small intestine by using the Cufflinks method for transcript assembly (see Additional file 1: supplementary methods). This analysis predicted six different RNA isoforms in this region, two of which correspond to Pint $A$ and Pint $B$ variants (see Additional file 1: Figure S2A). Pint $C$ was not detected by the RNA-seq analysis, which could be due to the different cell type used for RACE cloning. Interestingly, one of the experimentally validated p53REs (p53RE-1), is located at the 5 ' end of all detected transcripts, in agreement with the transcriptional activation of these response elements by p53 (see Additional file 1: Figure S2A). The other two p53 sites (p53RE-2 and p53RE-3) are respectively 100,000 and $120,000 \mathrm{bp}$ further downstream (Figure 1D). Additionally, ChIP-seq data from MEFs showed that p53RE-1 overlaps with a peak for H3K4me3, the chromatin mark associated with active promoters, while p53RE-2 and p53RE-3 are also enriched in H3K4me1 (see Additional file 1: Figure S2A), suggesting that the two distal sites could act as enhancers.

Next, to characterize the non-coding nature of the cloned RNAs, we analyzed the coding potential across all full-length isoforms identified. All the potential ORFs found in the transcripts are small $(<100$ amino acids) and do not contain evolutionary conserved codons (Codon Substitution Frequencies scores < -205), strongly suggesting a lack of protein-coding capacity [22].

We focused our studies on variant Pint A (1157 nt), which is the longest of the cloned isoforms and the one with the highest expression level (see Additional file 1, Figure S2B). Analysis of Pint A expression across a panel of normal mouse tissues showed that it is ubiquitously expressed (see Additional file 1: Figure S2C).

We next tested the expression of Pint in different mouse cell types at different time points after induction of $\mathrm{p} 53$ by doxorubicin-induced DNA damage, including $\mathrm{p}^{2} 3^{+/+}$and p53 ${ }^{-/-}$MEFs (Figure 1E), and K-RAS lung tumor and sarcoma cells (Figure 1F,G; see Additional file 1: Figure S2D) after genetic restoration of the $p 53$ gene [23]. In all cell lines tested, levels of Pint increased significantly in a temporal manner upon p53 induction.
Next, to further confirm the regulation of Pint by p53, we depleted p53 by small interfering RNA (siRNA) treatment in p53-restored $\mathrm{p} 53^{\mathrm{LSL} / \mathrm{LSL}} \mathrm{MEFs}$ (which are functionally equivalent to $\mathrm{p} 53^{+/+}$MEFs [23]), and transfected a non-targeting siRNA as control. Inhibition of p53 resulted in a decrease of Pint levels, whereas the control siRNA had no effect (see Additional file 1: Figure S2B). We therefore conclude that Pint expression is induced in a p53dependent manner.

Collectively, our results show that Pint is a ubiquitously expressed lincRNA, which has several isoforms and is transcriptionally regulated by $\mathrm{p} 53$.

\section{Pint modulates cell survival and proliferation}

To elucidate the biological role of Pint, we attempted to perform RNA interference (RNAi)-mediated loss of function studies. To that end, we designed multiple Pinttargeting siRNAs and short hairpin RNAs (shRNAs), and transfected or transduced cells to deplete the lincRNA levels. However, these strategies were unsuccessful in lowering the levels of Pint (data not shown), which prompted us to use an alternative approach.

We then designed anti-sense oligonucleotides (ASOs) with special modifications to target Pint for degradation by RNaseH [24]. By independently transfecting two different Pint-targeting ASOs we were able to obtain a significant decrease $(>75 \%)$ in Pint levels compared with transfections using two independent control ASOs or in the absence of oligo transfection (PBS) (Figure 2A; see Additional file 1: Figure S3A). Interestingly, we were able to deplete all three Pint isoforms (data not shown), supporting the notion that the inhibition by ASOs occurs at the level of pre-spliced RNA [25].

Next, to assess the effect of Pint downregulation, we treated p53-restored p53 ${ }^{\mathrm{LSL} / \mathrm{LSL}}$ MEFs with two independent Pint-targeting ASOs or with two independent control ASOs, and monitored cell proliferation at 24 and 48 hours after transfection, while treating the cells with doxorubicin to induce the p53 response (Figure 2B). There was a significant decrease in proliferation of cells depleted of Pint by the two specific ASOs compared with the cells treated with either of the two ASO controls or compared with the untransfected cells (Figure 2B). Conversely, when Pint (isoform A) was transiently overexpressed using a plasmid under the control of a cytomegalovirus promoter (Figure 2C), cell proliferation was increased compared with the cells transfected with the empty plasmid (Figure 2D). Similarly, stable overexpression of Pint by retroviral infection had a positive effect on cell proliferation rate (data not shown). Interestingly, we also found a slight effect on proliferation after Pint depletion and overexpression in the absence of doxorubicininduced DNA damage, although this was not as significant 
A

C
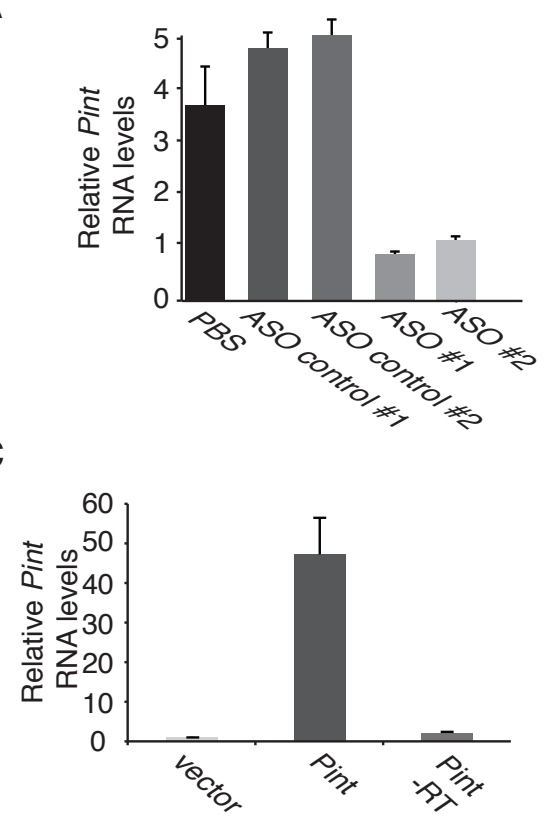

E

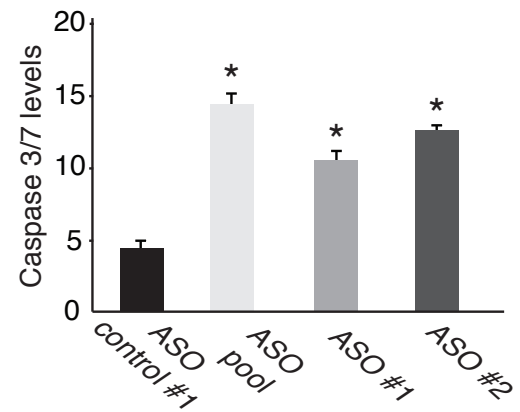

G

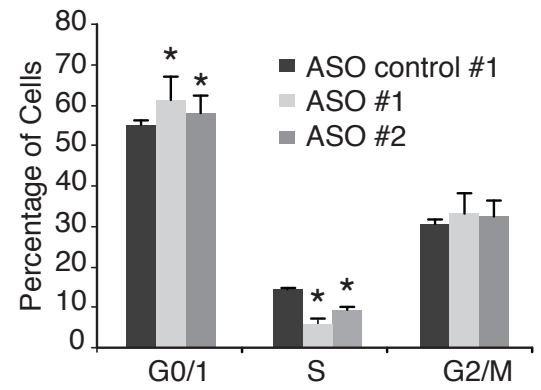

B

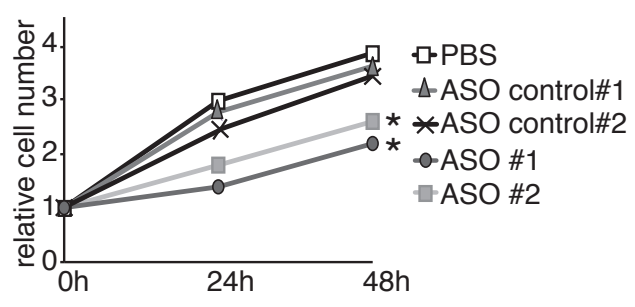

D

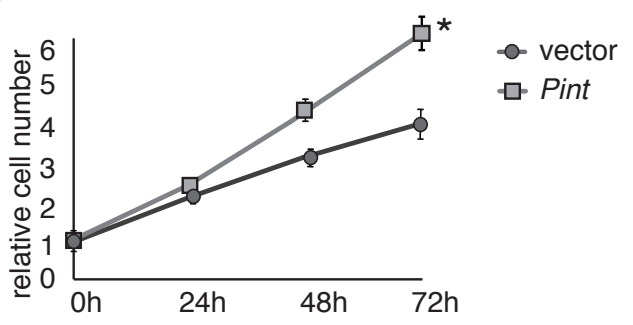

$\mathrm{F}$

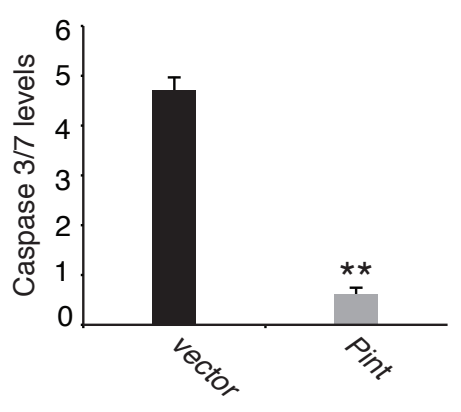

$\mathrm{H}$

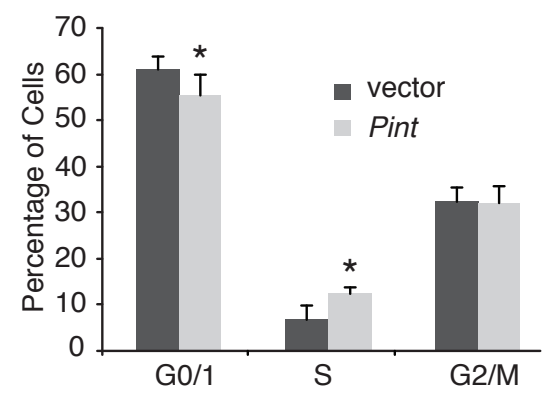

Figure 2 PINT modulates cell proliferation and apoptosis. (A) Inhibition of Pint. Pint levels were detected by quantitative real time (RT-qPCR) in p53-restored doxorubicin-treated p53 ${ }^{\text {LSL/LSL }}$ MEFs 36 hours after transfection with two Pint-specific anti-sense oligonucleotides (ASOs) (ASO1 and ASO2), two control ASOs (control ASO -1 and -2), or a blank (PBS) control, and 12 hours of doxorubicin treatment. Values normalized to Gapdh and are the mean \pm SD of three replicates. (B) Pint positively regulates cell proliferation. Relative number of p53-restored p53 ${ }^{\mathrm{LSL} / \mathrm{LSL}}$ mouse embryonic fibroblasts (MEFs) transfected with ASOs for Pint inhibition, and treated with doxorubicin from $24 \mathrm{~h}$ post-transfection. Cell numbers are determined by MTS assay. Values are mean \pm SD of three replicates. (C) Overexpression of Pint. Pint levels where measured like in (A) in p53-restored doxorubicin-

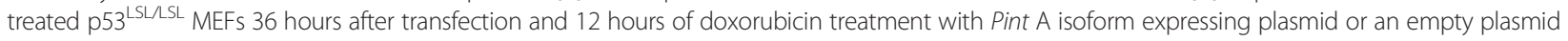
as control. (D) Pint positively regulates cell proliferation. Cells were transfected as in (C) and treated with doxorubicin from 24 hours post-transfection. $(\mathbf{E}, \mathbf{F})$. Negative effect of Pint on apoptosis induction. Apoptosis levels were determined by quantification of caspase 3/7 levels after (E) inhibition or (F) overexpression of Pint in p53-restored p53 ${ }^{\text {LSL/LSL }}$ MEFs treated with doxorubicin. Values are the mean \pm SD of three replicates. (G,H). Effect of Pint on cell cycle regulation. Relative cell numbers in each cell cycle phase were determined by fluorescence-activated cell sorting (FACS) of bromodeoxyuridine (BrdU) incorporation and propidium iodide (PI) staining of p53-restored p55 ${ }^{\text {LSLLSL }}$ MEFs treated as in (A) or (C). Percentages of cells in each phase are represented and values are the mean \pm SD of three replicates. 
as in the presence of doxorubicin (see Additional file 1: Figure S3B,C). We thus concluded that Pint is a positive regulator of cell proliferation.

To determine how cell proliferation is modulated by Pint, we investigated different aspects of the cellular phenotype. When Pint was depleted and cells were treated with doxorubicin to induce DNA damage, there was a significant increase in the number of apoptotic cells (Figure 2E). Consistent with these results, Pint overexpression resulted in the opposite phenotype, decreasing cellular apoptosis (Figure 2F). There was also a slight but significant effect on cell cycle regulation. Transfection of the specific Pint-targeting ASOs caused a decrease in the fraction of S-phase cells and a concomitant increase in the fraction of cells in G1 (Figure 2G), whereas Pint overexpression caused the opposite effect (Figure 2H). These data suggest that, under DNA-damaging conditions, Pint affects both induction of apoptosis and regulation of the cell cycle.

Next, to determine whether the aforementioned effects were cell type-specific, we performed similar experiments in the mouse lung tumor cell line LKR [3]. There was a significant decrease in cell proliferation following Pint depletion in these cells by doxorubicin-induced DNA damage (see Additional file 1: Figure S3D,E), whereas lincRNA overexpression caused the opposite effect (see Additional file 1: Figure S3F,G). Inhibition of Pint in doxorubicin-treated 3T3 cells caused a similar effect on cell proliferation and apoptosis induction (see Additional file 1: Figure $\mathrm{S} 3 \mathrm{H}$ to K). Furthermore, depletion of Pint in these cells affected their ability to grow independently of attachment and to form colonies independently of cell-cell contact (see Additional file 1: Figure S3L,M), opposite to the effect caused by Pint overexpression (see Additional file 1: Figure S3N).

We therefore concluded that Pint positively regulates cell viability and proliferation at different levels, including induction of cellular apoptosis and regulation of the cell cycle, both in the presence and absence of DNA damage.

\section{Pint regulates the expression of genes involved in cell proliferation and survival, including genes of the p53 pathway}

Given the role of Pint in cell survival and proliferation, we wanted to investigate the effect of the lincRNA on gene expression. We transfected p53-restored $\mathrm{p} 53^{\mathrm{LSL} / \mathrm{LSL}}$ MEFs with a pool of ASOs to deplete Pint expression or with a non-targeting ASO as control, then treated the cells with doxorubicin to induce DNA damage, and extracted total RNA for microarray analysis in triplicate. We identified 947 genes affected by lincRNA inhibition (B > 3) (see Additional file 1,: Figure S4A; see Additional file 3). Gene Ontology analysis of these genes identified significant enrichment in pathways relevant for signaling, proliferation, and survival, including extracellular matrix
(ECM)-receptor interaction and transforming growth factor (TGF)- $\beta$, mitogen-activated protein kinase (MAPK), or p53 signaling pathways (Figure 3A; see Additional file $3)$. In agreement with this observation, the most significant biological functions of Pint-regulated genes included cancer, cellular movement, cellular growth and proliferation, cell death and survival, and organism development (see Additional file 1: Figure S4B).

To independently validate the microarray findings, cells were transfected with two different Pint-targeting ASOs or two control ASOs, and the levels of 15 mRNAs were determined by quantitative real time (RT-qPCR). This experimental validation confirmed the microarray results for 14 of 15 genes (93\%), including the downregulation of $\operatorname{Tg} \beta \beta 1$, Serpina3n, Nkx2-9, and Il1r1, and upregulation of Gadd45b and Egr2, among others (see Additional file 1, Figure S4C).

Interestingly, genes affected by Pint inhibition did not include any of the six neighboring genes located $250 \mathrm{~kb}$ upstream or downstream of the Pint locus. Furthermore, upon Pint depletion, we did not observe any change in the levels of the microRNA mir29a/b, encoded downstream of Pint, which we determined by RT-qPCR on the small RNA fraction of cells (data not shown). Therefore, our data suggest that although Pint depletion affects the expression of hundreds of genes, Pint does not act on genes that are proximally located to it.

The direct transcriptional regulation of Pint by p53 strongly suggests a functional relationship between the two. This relationship was confirmed by the presence of the p53 pathway as one of the cellular pathways most affected by Pint inhibition (Figure 3A). Moreover, the microarray data analysis predicted p53 as one of the upstream regulators of genes affected by Pint $(\mathrm{B}>5, P=$ $4.20 \times 10^{-13}$ ) (Figure 3B; see Additional file 4).

To further explore the relationship between Pint and p53, we treated cells with p53 siRNA or a control siRNA, and subjected the extracted RNA to microarray analyses (Figure 3C). As expected, the analyses showed that hundreds of genes were affected by p53 depletion (1,146 genes, B > 3), including most of the well-known p53 target genes such as $C d k n 1 A$, Fas, and Perp (see Additional file 5).

Next, to detect genes co-regulated by p53 and Pint, we compared the genes affected by $\mathrm{p} 53$ depletion with those affected by Pint depletion. Interestingly, a significant subset of the genes affected by Pint inhibition was similarly affected by p53 inhibition ( 86 genes, $\mathrm{B}>3, P=1.5 \times 10^{-5}$ ) (Figure 3A,D). These genes were enriched in functional terms that include cellular apoptosis and cell cycle regulation (Figure 3A), and secondary targets of p53, such as Ikbke, Dgka, Adam8, and Serpine2 (see Additional file 6). These results confirmed that Pint gene regulation comprises part of the p53 transcriptional response.

In addition to the transcription factor p53, other upstream regulators are predicted for Pint-regulated 


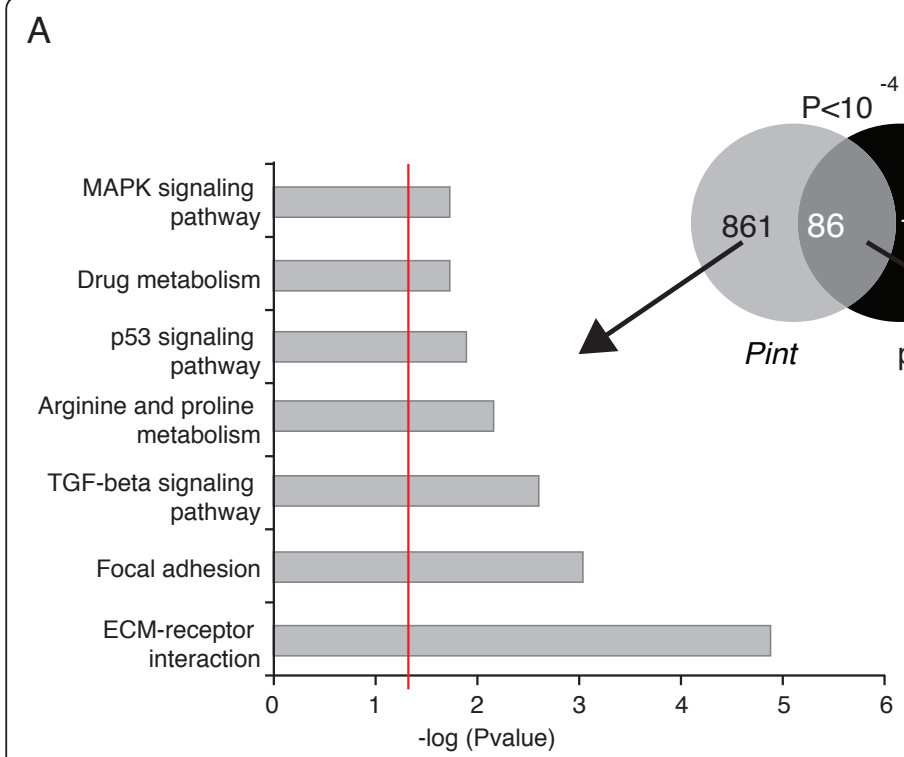

B
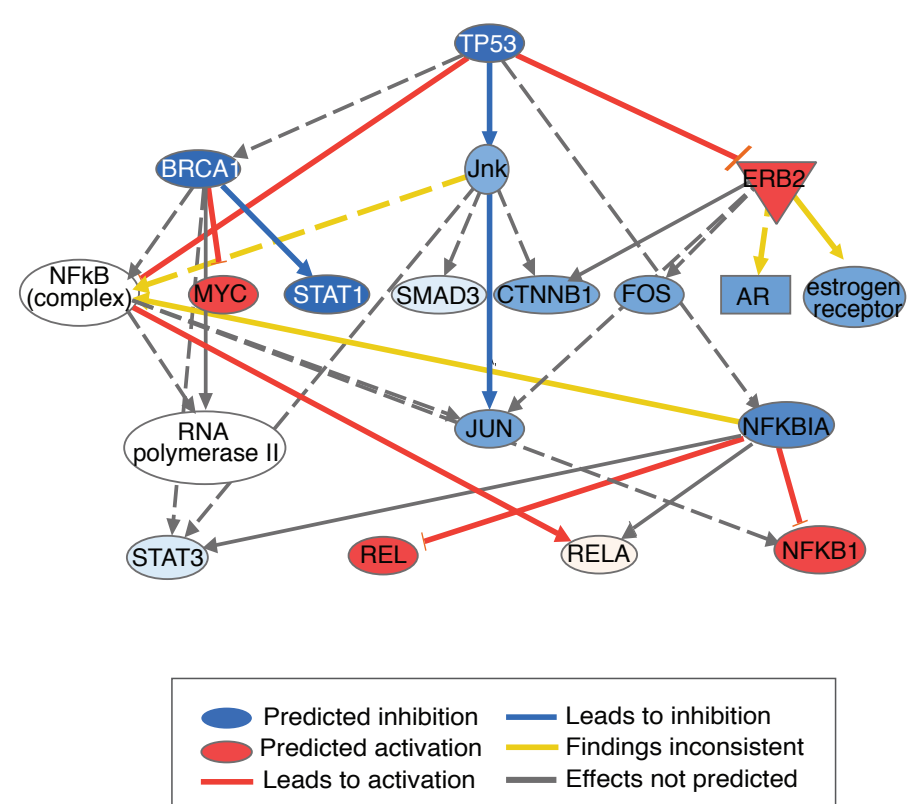
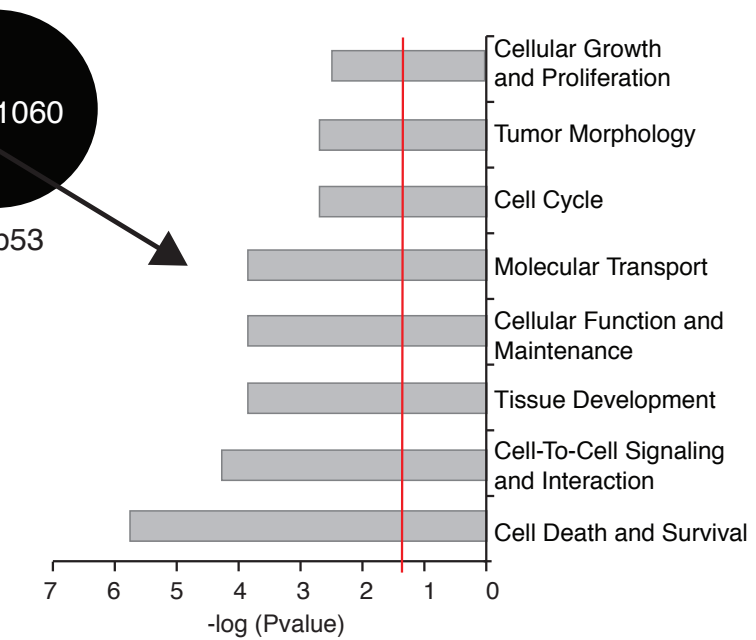

C

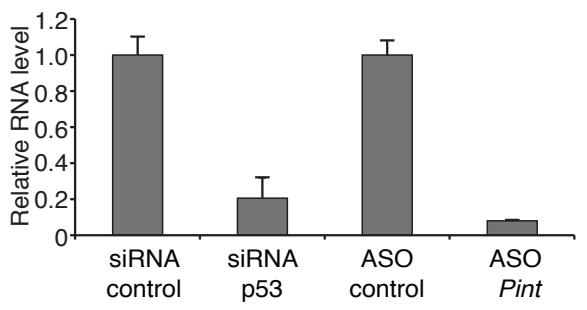

D

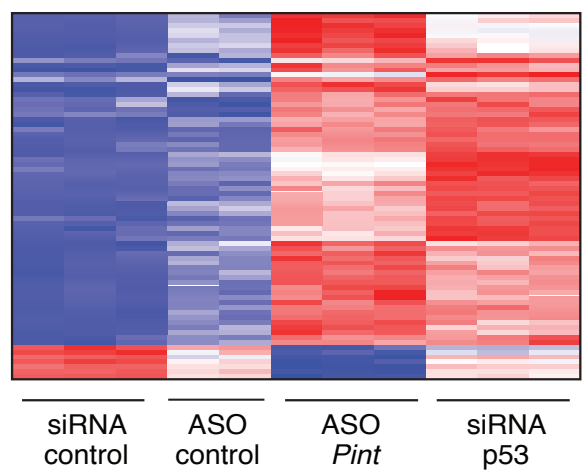

Figure 3 PINT regulates the expression of genes involved in cell proliferation and survival, including genes of the p53 pathway

(A) (Left) Significant KEGG (Kyoto Encyclopedia of Genes and Genomes) pathways enriched in the 947 genes regulated by Pint. Center: Venn diagram representing the number of genes affected by Pint (947), and p53 knockdown in p53-restored doxorubicin-treated p53 ${ }^{\text {LSL/LSL }}$ mouse embryonic fibroblasts (MEFs) $(B>3)$. P-value represents the probability associated with the overlap between both gene sets (86 genes). (Right) Significant biologic functions of genes co-regulated by Pint and p53. The red line represents $P=0.05$. (B) Predicted p53 regulatory network based on the fold change of genes affected by Pint depletion (ingenuity pathway analysis). (C) Relative Pint RNA levels after Pint or p53 depletion. Values are normalized to Gapdh and are the mean \pm SD of four replicates. (D) Genes commonly affected by Pint and p53 depletion $(B>3)$. Colors represent transcripts above (blue) or below (red) the global median, scaled to twofold activation or repression, respectively.

genes, including the cytokine tumor necrosis factor $\alpha$, the transcription regulator nuclear factor $\kappa \mathrm{B} 1 \mathrm{~A}$ or the tumor growth factor $-\beta 1$ (see Additional file 4). We therefore investigated the genes that are regulated by Pint but not p53. The most significant biological functions of the genes regulated specifically by Pint include cell death, response to hypoxia, and vasculogenesis (see Additional file 1: Figure S4D). By contrast, the top biological functions of the genes regulated by $\mathrm{p} 53$ but not Pint are segregation of chromosomes, mitosis, and cell cycle progression (see Additional file 1: Figure S4E). These results confirm that Pint is involved in biological processes related to survival 
and invasion, which are different from those of the p53 core response. In addition, the Pint-independent component of the p53 pathway is clearly enriched in cell cycle regulatory genes.

Taken together, our data strongly suggest that Pint plays an important role in gene regulation via a trans-mediated mechanism, modulating cellular pathways that are crucial for cell survival and proliferation, including genes of the p53 pathway.

\section{Pint, a nuclear RNA that interacts with Polycomb repressive complex 2}

We next investigated the mechanism by which Pint regulates gene expression. We first analyzed the subcellular localization of Pint by RT-qPCR in nuclear versus cytoplasmic fractions, and found that at least $80 \%$ of the Pint RNA was present in the cell nucleus (Figure 4A). We further confirmed this observation by single-molecule RNA fluorescence in situ hybridization (FISH) to detect individual molecules of Pint in 3T3 MEFs. The analysis showed that more than $85 \%$ of the PINT foci were present in the cell nucleus (Figure 4B,C).
Because many lincRNAs have been found to be associated with nuclear protein complexes $[7,8,10]$, we hypothesized that this could be the case for Pint. Interestingly, a transcript that we found to correspond to Pint was previously identified in a genome-wide screen by RNA immunoprecipitation sequencing (RIP-seq) for PRC2-interacting RNAs in mouse embryonic stem cells [26]. Moreover, analysis of the Pint A sequence revealed the presence of 10 Ezh2-interacting motifs [27], a larger number than would be expected by chance $(P<0.05)$. Therefore, we decided to test the interaction between Pint and PRC2.

To do so, we performed crosslinking followed by RIP in doxorubicin-treated MEFs using an antibody specific for the Suz12 subunit of PRC2 or, as control, an antibody against WD repeat domain 5 (Wdr5), a protein associated with the mixed lineage leukemia (MLL) chromatin activator complex. We found a highly significant enrichment of Pint in PRC2 immunoprecipitates, whereas no Pint enrichment was seen with the Wdr5 antibody or control IgG (Figure 4D).

To further confirm the interaction between Pint and PRC2, we carried out RNA pulldown experiments using in

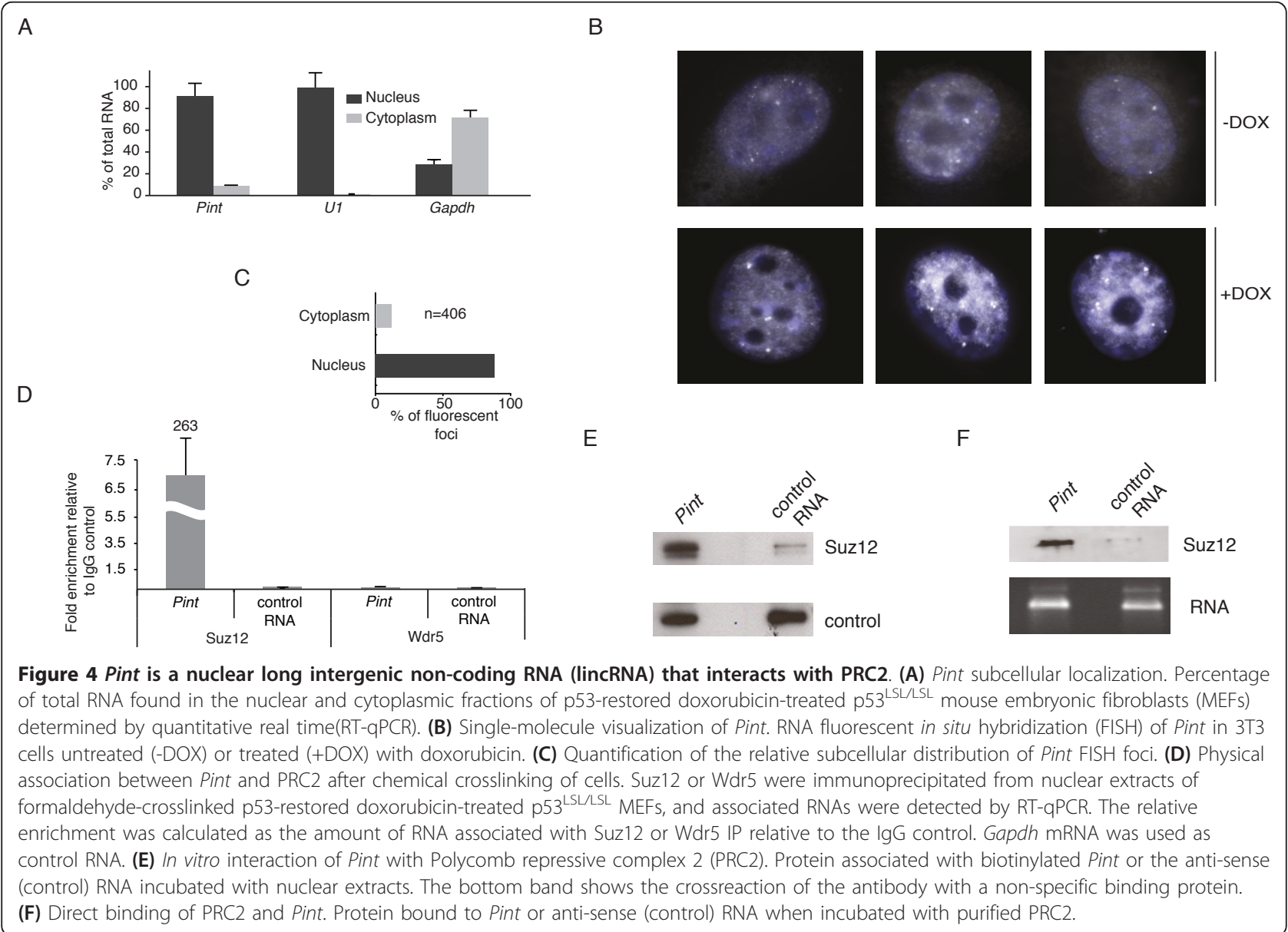


vitro synthesized and biotin-labeled Pint RNA, and nuclear extracts of doxorubicin-treated MEFs. Pint was able to pull down PRC2, as detected by western blotting using an anti-Suz12 antibody, whereas only negligible levels of Suz12 were bound by the anti-sense RNA sequence used as control (Figure 4E).

In addition, to determine whether the interaction between Pint and PRC2 was direct or mediated by other factors, we performed RNA pulldown experiments with in vitro biotinylated Pint RNA and purified PRC2. We found that Pint was able to interact with the recombinant purified PRC2, unlike the control RNA (Figure 4F), suggesting that Pint directly binds to PRC2.

In conclusion, our data show that Pint is mainly localized to the cell nucleus, and directly interacts with PRC2.

\section{Pint affects gene expression by regulating PRC2 occupancy of specific genes for repression}

Several studies suggest that the association of lincRNAs with chromatin complexes such as PRC2 provides regulatory specificity to the complexes by localizing them to genomic DNA targets $[3,10,11,28-30]$. We therefore hypothesized that Pint may act by regulating the binding of PRC2 to certain genomic loci for their repression. Consistent with this hypothesis, we found that a significant number of the genes regulated by Pint (141 genes, $P=1.4$ $\times 10^{-7}$ ) had been previously reported [31] as bound by PRC2 in mESCs (Figure 5A; see Additional file 7). In agreement with this observation, we found this subset of Pint-regulated genes to be enriched in H3K27 tri-methylation around their transcriptional start site, whereas the remaining Pint -regulated genes showed low H3K27me3 but high H3K4me3 levels (Figure 5B; see Additional file 1: Figure S5A). Interestingly, pathway analysis of these genes identified enrichment in MAPK signaling, ECM-receptor interaction, and TGF- $\beta$ signaling, consistent with these pathways being affected by Pint inhibition (see Additional file 1: Figures S5B and S3A). Furthermore, the most significant biological function of this gene subset is cellular growth and proliferation (Figure $5 \mathrm{C}$ ).

Next, to further test our hypothesis of Pint requirement for PRC2 targeting, we proceeded as follows. We randomly selected a group of 15 genes that (i) we found to be de-repressed by inhibition of Pint in p53-restored p53 ${ }^{\text {LSL/LSL }}$ MEFs (see Additional file 3; see Additional file 1: Figure $\mathrm{S} 5 \mathrm{C}$ ), and (ii) have been reported as regulated by $\mathrm{H} 3 \mathrm{~K} 27 \mathrm{me} 3$ in MEFs [32]. We reasoned that these genes might be co-regulated by PRC2 and Pint. We then determined the association of PRC2 with these genes by Suz12 ChIP-qPCR in p53-restored p53 ${ }^{\text {LSL/LSL }}$ MEFs, with or without inhibition of Pint (Figure 5D). The ChIP results confirmed that Suz12 occupied all the analyzed genes in the control conditions (see Additional file 1: Figure S5D). Interestingly, the binding of Suz12 to 12 of these loci (80\%) was significantly decreased upon Pint depletion (Figure 5D; see Additional file 1: Figure S5D), correlating with an increase in their expression (see Additional file 1: Figure S5C). However, there was no change in Suz12 occupancy of genes that are bound by Suz12 but whose expression was unaffected by Pint knockdown (Figure 5D, controls; see Additional file 1: Figures S5C, D).

Similarly, we performed ChIP to determine the level of H3K27me3 at these gene promoters under the same experimental conditions. Correlating with the loss of PRC2 binding, there was a significant decrease in H3K27me3 levels in 11 of the 15 (73\%) analyzed regions when Pint was inhibited (Figure 5E; see Additional file 1: Figure S5E), whereas there were no significant changes at the control genes. Interestingly, in some cases, the changes in H3K27me3 were not as pronounced as the loss of Suz12 binding, probably because of the need for cell division and/ or histone demethylase activity to erase the histone mark.

We therefore conclude that Pint is required for PRC2 targeting to these genes, which in turn affects their H3K27 methylation levels and expression.

We speculated that if regulation by Pint is mediated by its interaction with $\mathrm{PRC} 2$, the effect of Pint in cell proliferation should be strongly dependent upon PRC2 presence. To test this hypothesis, we generated 3T3 MEFs with stable knockdown of the Ezh2 subunit of PRC2, using shRNA lentiviral transduction, and, as control, we transduced 3T3 MEFs with a non-targeting shRNA. The Ezh2 shRNA stable cell line showed a decrease of around $60 \%$ in Ezh2 protein levels compared with the shRNA control cell line (see Additional file 1: Figure S5F).

Next, we transfected both cell lines with a pool of ASOs to deplete Pint levels, or with a control ASO (see Additional file 1: Figure S5G,H), and determined their proliferation rate. The proliferation of control cells was negatively affected by Pint inhibition (Figure 5F), and interestingly, arrest of proliferation was strongly enhanced when both Pint and Ezh2 were depleted in the cells (Figure 5G). In parallel, both cell lines were transfected with a plasmid overexpressing Pint or a control plasmid, and their proliferation rate was determined. Although Pint overexpression induced proliferation of the control cells, it had no effect on the Ezh2-depleted cells (Figure 5F,G). These results suggest that the biological function of Pint requires PRC2, indicating a functional relationship between Pint and Ezh2.

Taken together, these data indicate that Pint is required for the targeting of PRC2 to some genes for repression, which in turn affects the proliferative state of the cells.

\section{Human PINT is a putative tumor suppressor lincRNA}

The role that Pint plays in gene regulation and in the p53 tumor suppressor pathway motivated us to explore 


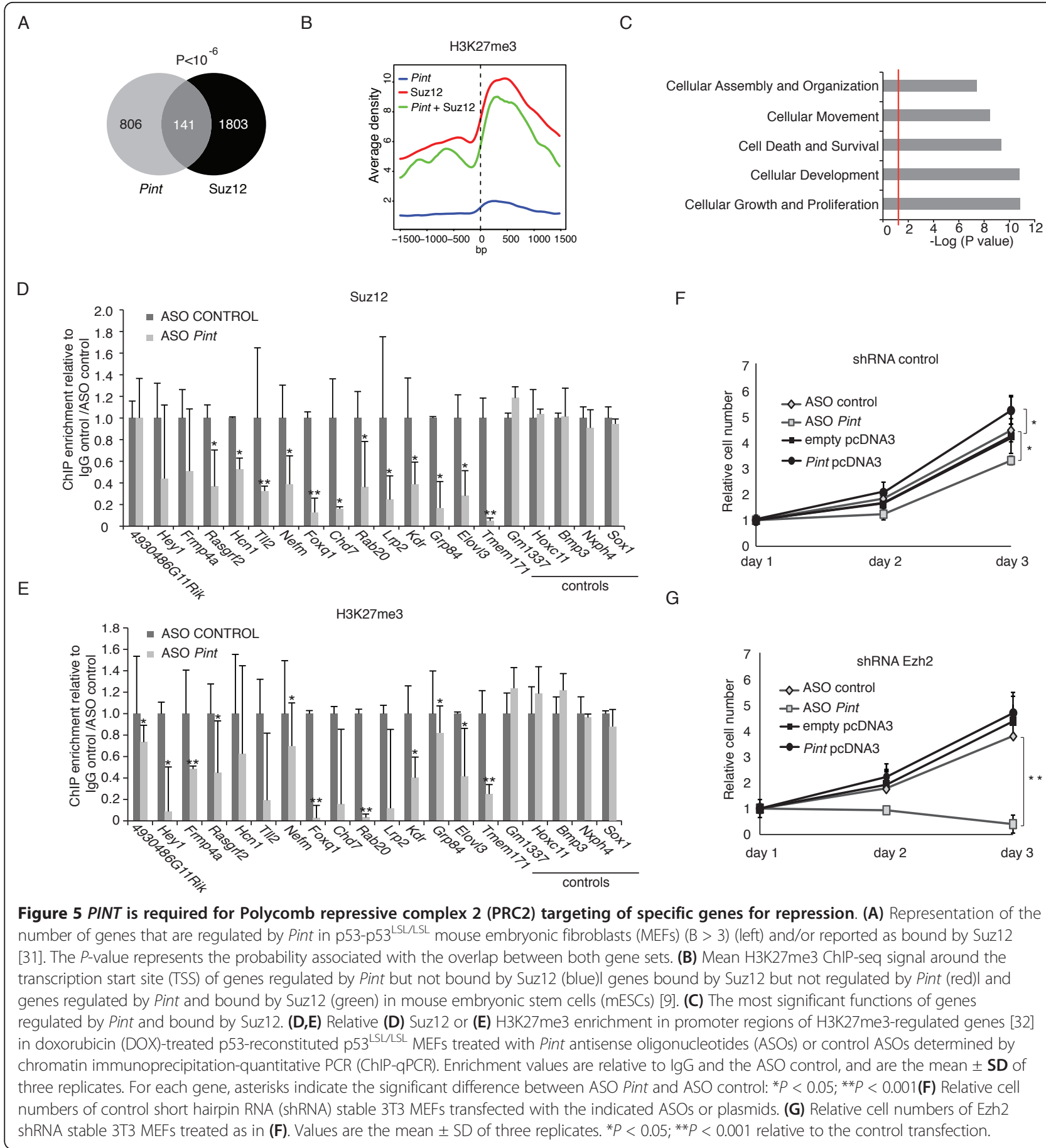

whether a Pint human ortholog exists. We found that the PINT human syntenic genomic region in chromosome 7 also encodes a non-coding RNA annotated as FLJ43663. Comparison of human and mouse sequences identified that the highest homology between the two lincRNAs resides at the 5 ' end of their sequences (see Additional file 1: Figure S6A,B). We therefore hypothesized that, similarly to mouse Pint, human PINT is regulated by p53.
To test this, we first analyzed the expression of PINT by qRT-PCR in $\mathrm{p}^{2} 3^{+/+}$and $\mathrm{p} 53^{-/-}$matched HCT116 human colorectal cancer cell lines [33], and found that PINT was induced in $\mathrm{p} 53^{+/+}$, but not $\mathrm{p} 53^{-/-}$cells when treated with the DNA-damaging drug 5-fluorouracil (5-FU) (Figure 6A, B). Next, to determine the functionality of the three conserved p53REs identified in the mouse and human PINT (see Additional file 2), we performed p53 ChIP on the 
human cells. The p53 protein was found to bind to the three human p53REs upon doxorubicin-induced DNA damage, but not in the absence of treatment (Figure 6C). As control, we also included p $53^{-1-}$ cells, in which we did not detect any p53 ChIP enrichment (Figure 6C).

To further confirm the regulation of PINT by p53, we cloned the human genomic sequences harboring each of the three p53REs into a plasmid containing a reporter gene. The three sequences were able to induce expression of the reporter gene when transfected into p53 wild-type, but not p53-null cells (Figure 6D).

Together, our data indicate that PINT is a bona fide p53 transcriptional target, with conserved regulation across mammalian species.

Given the crucial role that p53 plays in cancer, we speculated that PINT expression might be altered in primary tumors. To test this hypothesis, we analyzed PINT levels in tumor tissue and matched normal tissue samples from 14 surgical patients with colorectal cancer (stages I to III) (see Additional file 8). Intriguingly, we found a significant $(P<0.01)$ downregulation of PINT in colorectal tumors compared with normal tissue (Figure 6E), suggesting a potential role of the lincRNA as a tumor suppressor.

To explore this hypothesis, we investigated in more detail the role of PINT in human cells. We first stably overexpressed PINT in the HCT116 colon cancer cell line by retroviral infection (see Additional file 1: Figure S6C) and assessed the proliferation rate. Interestingly, cells overexpressing PINT showed a significant decrease in their growth rate compared with the control cells in either the presence or absence of doxorubicin-induced DNA damage (Figure 7A,B). This decrease in proliferation was confirmed by the cell cycle profile analysis. Compared with control cells, PINT -overexpressing cells had a lower percentage of cells in $\mathrm{S}$ phase in the absence of doxorubicin-induced DNA damage (Figure 7C), whereas upon doxorubicin treatment, PINT-overexpressing cells showed more pronounced cell cycle arrest, appearing as a significantly lower number of cells in $S$ phase and higher number in G1/0 and G2/M phases (Figure 7C). Subsequently, we quantified the apoptosis levels in these stable cell lines, and found increased apoptosis when PINT was overexpressed both in the presence and absence of doxorubicin-induced DNA damage (Figure 7D). We concluded that PINT has a negative effect on proliferation and survival of HCT116 cells.

Next, to test whether the observed effects of PINT overexpression are specific to HCT116 cells, we generated a stably PINT-overexpressing A549 lung adenocarcinoma cell line (see Additional file 1: Figure S6D), and subjected the cells to similar analyses, determining their proliferation rate, cell cycle profile, and apoptosis levels. Overexpression of PINT in A549 cells caused decreased proliferation (Figure 7E,F) with an increased number of cells arrested in G2/M phases, both in the presence and absence of doxorubicin-induced DNA damage, but being more pronounced with doxorubicin treatment (Figure 7G) Similarly, enforced PINT expression in A549 cells resulted in increased apoptosis, both in the presence and absence of doxorubicin treatment (Figure 7H). Together, these results indicate that PINT is a negative regulator of cell proliferation and apoptosis of tumor cells, which is consistent with a role as a tumor suppressor lincRNA.

Next, to obtain further insight into the role of PINT, we determined the biological pathways that are associated with PINT expression in normal tissues, where it has higher expression. To that end, we obtained gene-expression data from microarrays performed on 23 samples from normal colon $(n=14)$ and rectum $(n=8)$, and a normal colon cell line. The microarrays contained 60,000 probes designed to detect the expression of 27,958 protein-coding genes and 7,419 non-coding RNAs. We selected expression data from nine probes corresponding to PINT, and computed the correlation existing between these and the expression of mRNAs grouped in KEGG (Kyoto Encyclopedia of Genes and Genomes) pathways [34]. The analysis showed that PINT expression had a significant positive $(r>0.5, n=23, p<0.05)$ or negative $(r<-0.5, n=23 p<$ $0.05)$ correlation with a number of pathways similar to those regulated by its mouse ortholog, including the MAPK, Wnt, and TGF- $\beta$ pathways (positive correlations). and p53, apoptosis, and peroxisome proliferator-activated receptor signaling (negative correlations) (Figure 7I). Thus, our data suggest that PINT expression and regulation are conserved between mouse and human. However, their function results in different biological outcomes, possibly reflecting species-specific aspects of cellular pathways.

Collectively, our results show that PINT is a lincRNA specifically regulated by p53 in mouse and human cells. In mouse cells, PINT promotes proliferation and survival, and functions by regulating targeting of PRC2 to specific genes for repression. The human ortholog, PINT, is also transcriptionally regulated by $\mathrm{p} 53$, and its expression correlates with similar cellular pathways to those of the mouse lincRNA. However, in contrast to the mouse Pint, human PINT is a negative regulator of proliferation and survival, and is downregulated in colon cancer, representing a novel tumor suppressor candidate lincRNA.

\section{Discussion}

Although thousands of lincRNAs have been identified in mammalian cells, understanding of lincRNA biology and role in disease remains relatively poor. A common feature of lincRNAs is their fine transcriptional regulation [35,36], which may be key to their specific regulatory roles. The transcription factor $\mathrm{p} 53$ has been subjected to thorough scrutiny over the years because of its relevance in cellular 
A

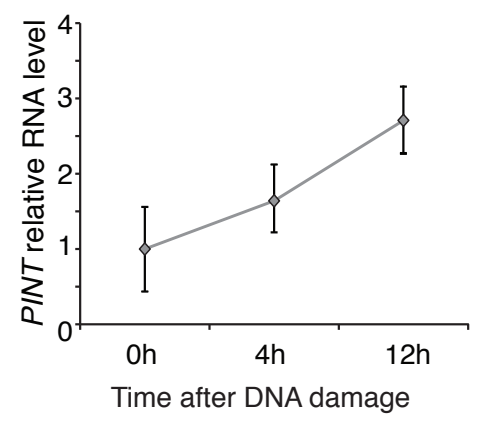

C

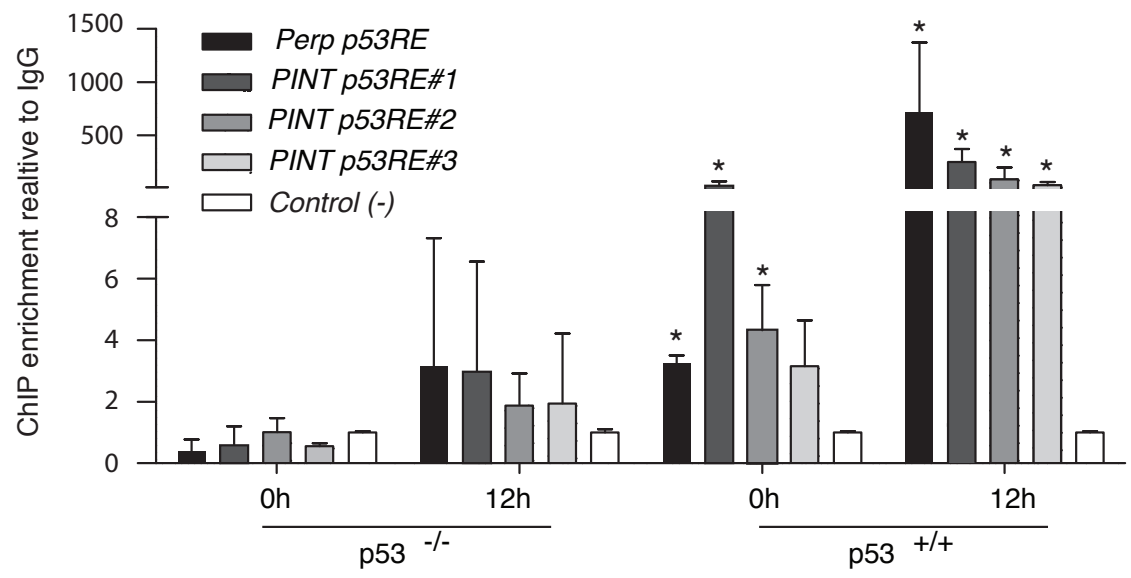

$E$
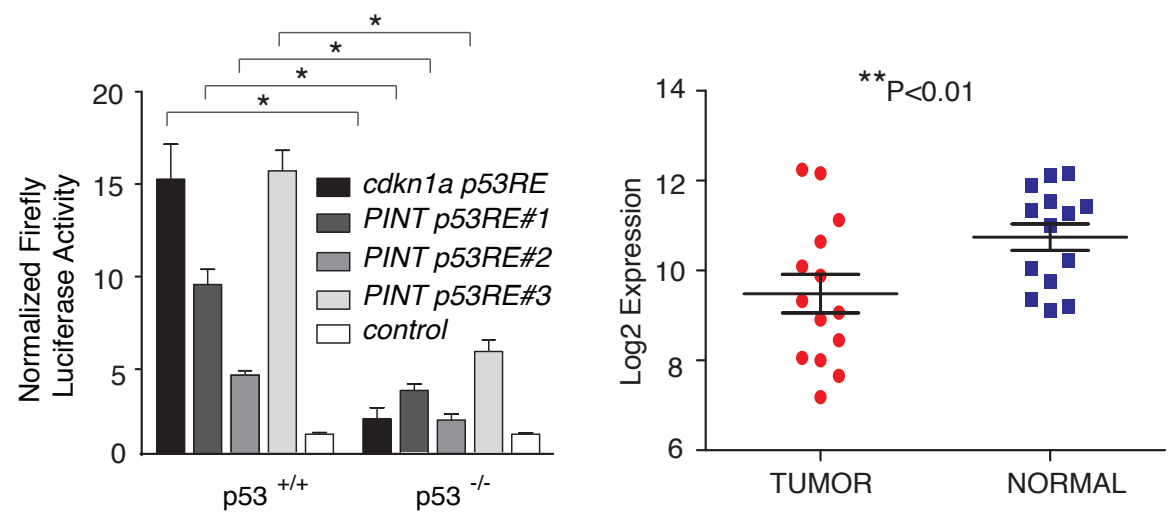

Figure 6 Human PINT is a p53-regulated long intergenic non-coding RNA (lincRNA) downregulated in colorectal cancer. (A) PINT is induced by 5-fluoracil (5-FU)-induced DNA damage. Relative PINT expression levels in HCT116 cells treated with 5-FU for the indicated times. (B) PINT is regulated by p53. Relative PINT levels in $\mathrm{p} 53^{+/+}$or $\mathrm{p} 53^{-/-}$matched HCT116 cells treated with 5 -FU for 12 hours. Values are the mean \pm SD of three replicates normalized to GAPDH. Asterisks represent significant difference between conditions. (C) p53 binds to p53 response elements (p53REs) in the PINT locus upon 5FU-induced DNA damage. Relative chromatin immunoprecipitation (ChIP) enrichment of p53 to the indicated regions in $\mathrm{p} 53^{+/+}$or $\mathrm{p} 53^{-/-}$matched HCT116 cells after the indicated times of treatment with 5-FU. Binding to the PERP promoter was included as a positive control and binding to an irrelevant genomic region as the negative control. Enrichment values are relative to IgG and to the negative control for each treatment condition. The mean \pm SD of three biologic replicates of a representative experiment is shown, and the significant differences relative to the control are indicated with asterisks. (D) p53 drives the transcription of PINT. Relative firefly luciferase expression driven by the indicated genomic sequences with p53REs in $\mathrm{p} 53^{+/+}$or $\mathrm{p} 53^{-/-}$matched HCT116 cells after treatment with 5 -FU. Values were normalized to Renilla levels and are the mean \pm SD of three replicates.(E) PINT is downregulated in colorectal tumors. PINT relative expression levels in colorectal cancer samples and normal peripheral tissue. 
A

C
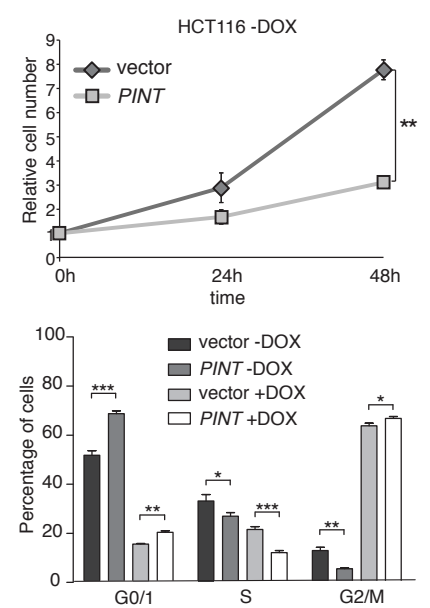

E

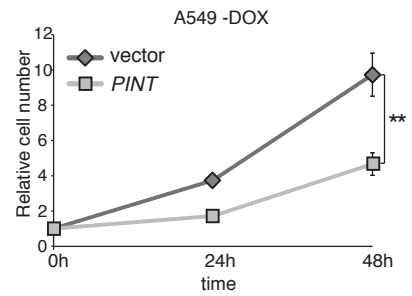

G

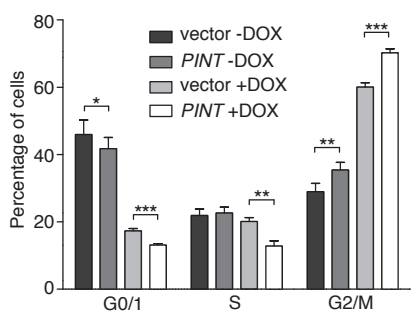

I
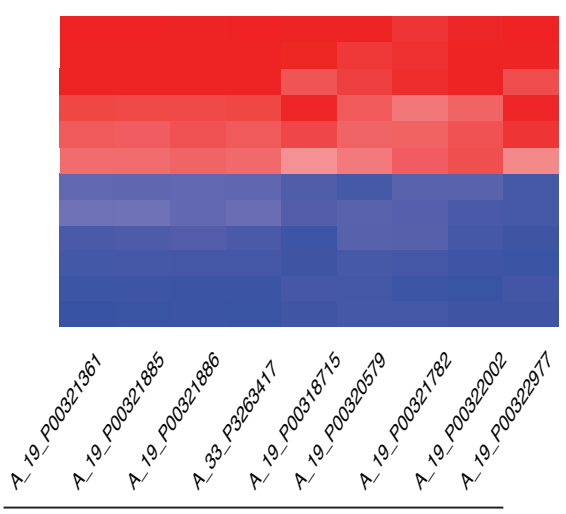

PINT probes
B

D
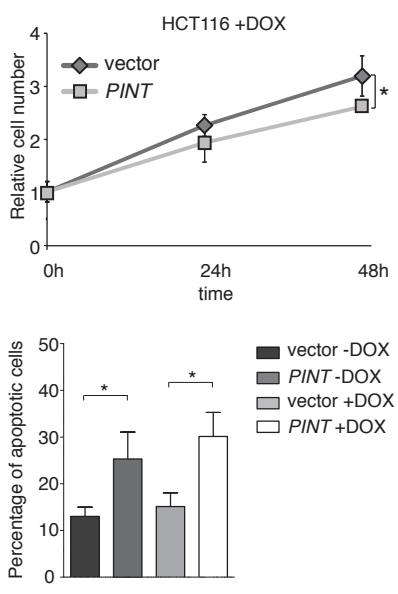

F

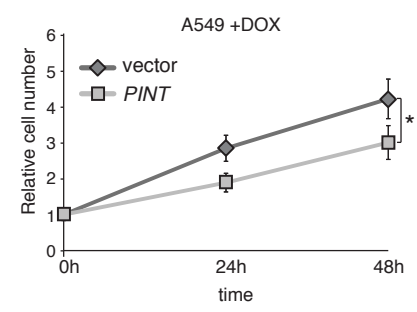

$\mathrm{H}$

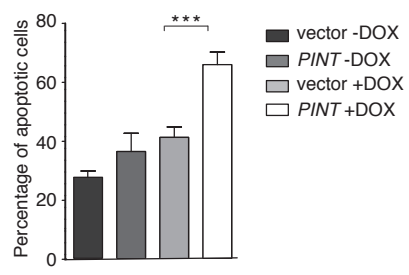

PATHWAYS

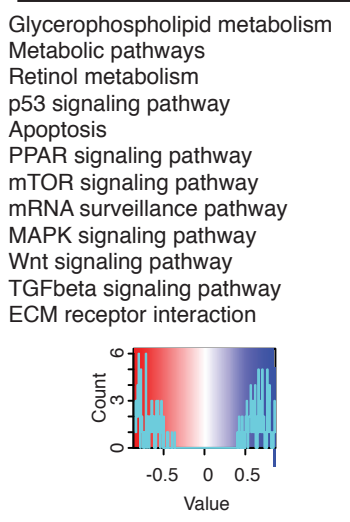
Metabolic pathways

Retinol metabolism

553 signaling pathway

Apoptosis

TGFbeta signaling pathway

ceptor interaction

Figure 7 Human PINT inhibits tumor cell growth. (A.B). Relative number of PINT-overexpressing HCT116 cells (PINT) or control cells (vector) that were (A) left untreated or (B) treated with $500 \mathrm{nM}$ doxorubicin (DOX), as determined by MTS assay. (C) Relative number of cells of HCT116 stable cell lines in each phase of the cell cycle. Cells were treated as for (A) and (B) for 12 hours, and cell cycle phases were determined by fluorescence-activated cell sorting (FACS) of bromodeoxyuridine (BrdU) incorporation and propidium iodide (PI) staining. (D) Percentage of apoptotic cells in HCT116 stable cell lines treated as in (C), determined by quantification of annexin V-positive cells. (E,F). Relative number of PINT-overexpressing A549 cells (PINT) or control cells (vector) that were (E) left untreated or were treated with 500 nM doxorubicin (F), as determined by MTS assay. (G) Relative number of A549 stable cell lines in each phase of the cell cycle determined as in (C). (H) Relative number of cells of A549 stable cell lines undergoing apoptosis, determined as in (D). (I) Correlation of PINT with KEGG (Kyoto Encyclopedia of Genes and Genomes) pathways. Significant correlation coefficients between the indicated cellular pathways and PINT microarray probes. 
homeostasis, but only recently have researchers realized that lincRNAs are an active part of the p53 transcriptional network. Among them is lincRNA-p21, which functions as a transcriptional gene repressor in mouse cells [3], and PANDA, which regulates the expression of pro-apoptotic genes in human fibroblasts [4]. Additionally, the lncRNAs linc-RoR [37] and loc285194 [38] have been reported to be post-transcriptional regulators in this pathway. In this study, we have expanded this knowledge by identifying and characterizing Pint, a bona fide p53 transcriptional target that acts as negative modulator of the p53 response.

We identified three isoforms of Pint, transcribed from an intergenic region in the mouse chromosome 6 . These transcripts are likely to be alternatively spliced variants, as they all share the 5 ' sequences and are regulated by $\mathrm{p} 53$. Indeed, Pint transcription is closely controlled by $\mathrm{p} 53$, which specifically binds to three functional p53REs contained inside the Pint genomic locus. While one of the three p53REs is located at the promoter, the other two are several kilobases downstream, and could function as transcriptional enhancers, contributing to the fine regulation of Pint levels upon p53 activation.

Pint levels are finely controlled by p53, but unlike many other known lincRNAs [36], Pint is ubiquitously expressed. Even in the absence of p53 activation, Pint is relatively robust (see Additional file 1: Figure S2C). This suggests that Pint plays a role independently of p53 activation by DNA damage. In fact, inhibition of Pint in the absence of DNA damage causes an arrest in cell proliferation, as opposed to the effect of Pint overexpression. Furthermore, even in the presence of DNA damage, most of the genes found to be regulated by Pint are involved in cellular pathways not directly related to p53. This suggests that Pint is necessary for regulation of normal cell growth and proliferation, and in the presence of DNA damage, Pint acts as a negative regulator of cell cycle arrest and as a pro-survival molecule, modulating the effect of p53 activation through a negative autoregulatory mechanism.

Pint binds directly to PRC2, and is required for the targeting of PRC2 to specific genes for H3K27 tri-methylation and repression. The association of PRC2 with the promoter of these genes is lost when Pint is depleted from the cells, resulting in their transcriptional induction. Moreover, the biological effect of Pint depletion is strongly enhanced by PRC2 downregulation, whereas Pint overexpression has no effect in a PRC2 knockdown background. These results suggest that Pint cooperates with PRC2 in the repression of genes required for survival and proliferation.

We found that of the total number of genes affected by Pint inhibition, 39\% were upregulated and 61\% downregulated upon Pint knockdown, suggesting that many Pintregulated genes are indirect targets of Pint-induced gene repression Interestingly, the top functional terms of the genes downregulated by Pint are related to transcription regulation (transcription regulator activity, transcription factor activity, and DNA binding). By contrast, Pint-upregulated genes are mostly involved in functions related to extranuclear components of signaling cascades such as pattern binding and polysaccharide binding (see Additional file 1: Figure S4F,G). These data are consistent with a model in which Pint modulates the targeting of PRC2 to specific transcription regulators, affecting the gene-expression cascade at its top, and resulting in broad downstream effects.

The precise mechanism by which Pint contributes to PRC2 targeting to specific loci remains to be defined. One possibility is that Pint binds to genomic sequences, either by Crick-Watson base pairing or DNA-RNA-DNA triple helical structures. The latter has been shown for other noncoding RNAs, resulting in transcriptional repression in the case of the dihydrofolate reductase gene, DHFR [39], or in epigenetic silencing in the case of ribosomal genes [40]. Additionally, we cannot exclude the possibility that Pint interacts with protein complexes other than PRC2,, acting as an RNA scaffold that brings together additional factors that may determine target specificity. In fact, this has been shown for the lincRNA HOTAIR, which interacts with the PRC2 and Lysine-specific demethylase 1 complexes [41].

We have identified the human PINT ortholog, which, despite relatively low overall sequence homology, shows several analogies with mouse lincRNA. Human PINT is not only transcriptionally induced by $\mathrm{p} 53$, but it conserves all three fully functional p53REs present in mouse. PINT significantly correlates positively or negatively with the same KEGG pathways that are affected by Pint knockdown in mouse cells. Furthermore, similarly to the mouse Pint, human PINT presents nuclear localization, and has previously been reported to interact with PRC2 [10]. The similarities between murine and human lincRNAs suggest that their study could help infer the molecular principles underlying lincRNA functions with low sequence dependency. Intriguingly, PINT appears to be significantly downregulated in primary colon tumors, and its overexpression in human tumor cells inhibits their proliferation. These observations contradict what might be expected based on a simplistic interpretation of the mouse in vitro phenotype, and could reflect species-specific aspects of cellular pathways and/or the known intrinsic biological differences between mouse in vitro models and human tumor cells [42].

\section{Conclusions}

In summary, we have identified a lincRNA, Pint, which establishes a new connection between the tumor suppressor p53 and epigenetic regulation by PRC2. Furthermore, 
the human ortholog of Pint may represent a crucial component of the p53 barrier against cancer.

\section{Materials and methods}

Cell lines, p53 restoration, and DNA damage induction

Lung tumor-derived cell lines were derived from individual tumors in KrasLA2/+;Trp53LSL/LSL Rosa26CreERT2 animals [43]. Sarcomas were isolated when they formed in Trp53LSL/LSL Rosa26CreERT2 animals as described previously [23]. p53 ${ }^{\mathrm{LSL} / \mathrm{LSL}} \mathrm{MEFs}$ were isolated from embryos of the same mouse strain. For p53 restoration, cultured tumor cell lines were incubated with 500 nM 4-hydroxytamoxifen (Sigma) for the indicated time points, and $\mathrm{p} 53^{\mathrm{LSL} / \mathrm{LSL}}$ MEFs were infected with AdenoCre or AdenoGFP virus for 24 hours (at the University of Iowa) at a multiplicity of infection (MOI) of 5. NIH/ 3T3 MEF cells were purchased from ATCC. The LKR Lung tumor-derived cell line was isolated from individual tumors from KrasLA2/+ mice. HTC116 $\mathrm{p}^{+/ /+}$and $\mathrm{p} 53^{-/-}$ were kindly provided by Dr Vogelstein's laboratory. For DNA damage, cells were treated with 100 to $500 \mathrm{nM}$ doxorubicin hydrochloride (D1515; Sigma) or $385 \mu \mathrm{M}$ of 5-FU.

\section{Promoter reporter assays and chromatin immunoprecipitation}

PINT genomic sequences (about 2,000 bp) flanking p53REs were amplified from human and mouse genomic DNA, and subcloned into pGL3-basic vector (Promega). The TK-Renilla plasmid was used as normalizing control. Firefly and Renilla luciferase activities were measured using the dual luciferase reporter assay kit (Promega) and a FLUOstar Optima luminometer (BMG Labtech). ChIP experiments were performed as previously described [44].

\section{Rapid amplification of CDNA ends (RACE)}

Using TRIzol reagent, total RNA was isolated from NIH/ 3T3 MEFs treated with doxorubicin hydrochloride for 12 hours. cDNAs were then amplified, and PINT isoforms were identified using the First Choice RLM-RACE Kit (Ambion), and followed by DNA sequencing.

\section{Stable cell line generation}

For stable PINT overexpression, PINT was cloned into the $\mathrm{pBABE}$ vector for retrovirus production, then NIH/ 3T3 MEFs were infected and selected with $1.5 \mu \mathrm{g} / \mathrm{ml}$ of puromycin for $72 \mathrm{~h}$. For generation of Ezh2 shRNA and stable control NIH/3T3 cells, shRNA lentiviral infection was used as previously described [45].

\section{qPCR primers and Antibodies}

The qRT-PCR and ChIP-qPCR primer sequences and antibodies used in this study are listed (see Additional file 1: Supplemental methods).
siRNAs, anti-sense oligo transfection, and PINT transient overexpression

All siRNAs and ASOs used in this study are listed (see Additional file 1: Supplemental methods). All ASOs were designed and provided by ISIS Pharmaceuticals. All were 20 nucleotides in length and were chemically modified with phosphorothioate in the backbone, five 2'-O-methoxyethyl residues at each terminus, and a central deoxynucleotide region of 10 residues (5-10-5 gapmer). ASOs were synthesized using an Applied Biosystems 380B automated DNA synthesizer (PerkinElmer Life and Analytical SciencesApplied Biosystems), and purified as previously described [24]. ASOs were selected from a larger panel of oligos, based on the achieved PINT RNA inhibition and an absence of toxicity. ASOs were used independently (ASO-1 and ASO-2) or as a pool (ASO-1 to ASO-4) (knockdown levels for each ASO independently are shown; see Additional file 1: Figure S3A). In all cases, ASOs and siRNAs were transfected at a total concentration of $100 \mathrm{nM}$ with Lipofectamine 2000 (Invitrogen) following the manufacturer's instructions. For transient overexpression experiments, PINT sequence was cloned into pcDNA3.

\section{Microarray analysis}

For gene-expression profiling, total RNA was extracted and hybridized to an Affymetrix Mouse Genome 4302.0 microarray. For human tissue samples, total RNA was hybridized to Agilent SurePrint G3 8x60K microarrays. Data normalization and analysis were performed with GiTools [34] (for more information see (see Additional file 1: Supplemental methods)

\section{Cell proliferation assays}

For proliferation analysis, 2,000 cells were plated per well in 96-well plates and assessed with a CellTiter96 Aqueous Non-Radioactive Cell Proliferation Assay (MTS) Kit (Promega).

For clonogenicity assays, cells were transfected, plated at $10^{3}$ to $2 \times 10^{3}$ cells per well of a six-well plate and grown in normal medium for 10 days. Cells were then fixed and stained with crystal violet. For soft agar colony formation assays, $1 \times 10^{4}$ and $5 \times 10^{4}$ cells $/ \mathrm{ml}$ were plated in a volume of $1 \mathrm{ml} \mathrm{0.3 \%}$ agar (Ref. 214220, C-35; DIFCO)

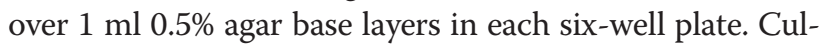
tures were monitored for growth by viewing under an inverted microscope. At the time of maximum colony formation (7 to 21 days of culture), colonies were stained with MTT (Sigma), and digital photgraphs were taken.

\section{Apoptosis and cell cycle analyses}

At 24 hours after transfection, $1 \times 10^{5}$ cells were plated in 96-well white microplates, and treated for 24 hours with $500 \mathrm{nM}$ doxorubicin. Apoptosis was determined by quantification with caspase-Glo 3/7 reagent (Promega) using a FLUOstar Optima luminometer, and with annexin 
V fluorescence-activated cell sorting (FACS) with an Apoptosis Detection Kit I (cat-559763; BD Biosciences). For cell cycle analysis, cells were labeled for 3 hours with bromodeoxyuridine (BrdU), and stained with propidium iodide (PI) using a BrdU flow kit(BD Bioscience) and sorted and quantified with a BD FACSCalibur flow cytometer (BD Biosciences). Data represent the mean \pm SD of a minimum of three biological replicates.

\section{Human samples}

Samples from patients with colorectal cancer (tumor and normal tissue; see Additional file 8) were obtained by surgical resection at the Municipal Hospital of Badalona, Spain. Tumors were staged in accordance with the American Joint Cancer Committee (AJCC) criteria. The adjacent normal tissue was obtained from areas $20 \mathrm{~cm}$ distant from the tumor, and diagnosis of normal mucosa was confirmed histologically. The work was carried out in compliance with the Declaration of Helsinki, and all patients provided signed informed consent.

\section{Nuclear fractionation and fluorescence in situ hybridization}

Nuclear fractionation was performed as previously described [3]. RNA FISH for PINT detection was performed using a pool of 48 fluorescent probes purchased from Stellaris Biosearch Technologies, following the manufacturer's protocol.

\section{X-linked RNA immunoprecipitation and RNA pulldown} RNA immunoprecipitation was performed after formaldehyde crosslinking of cells, as described previously [3]. RNA pulldowns were performed as described previously [28]. PRC2 was purchased from BPS Bioscience (catalogue number 3m:51003)

\section{Statistical analysis}

Experimental data are represented as the mean \pm SD of a minimum of three biologic replicates and were compared using Student's $t$-test. Significant $P$-values are indicated with asterisks as follows: ${ }^{*} P<0.05,{ }^{* *} P<0.01$, and ${ }^{* * *} P<0.001$.

\section{Accession numbers}

Full-length sequences of PINT A, B and C have been deposited in GenBank (accession numbers KC860257, KC860259, KC860258 respectively). All primary data are available at the Gene Expression Omnibus (GSE46272).

\section{Additional material}

Additional file 1: Supplemental materials and methods, and supplementary figures with figure legends and PINT sequences.
Additional file 2: Table S1. p53 response elements (p53REs). found in mouse and human PINT genomic loci.

Additional file 3: Table S2 Genes affected by Pint inhibition. Additional file 4: Table S3 Predicted upstream regulators of genes affected by Pint knockdown.

Additional file 5: Table 54 Genes affected by p53 inhibition.

Additional file 6: Table S5 Genes commonly affected by Pint and p53 inhibition.

Additional file 7: Table S6 Genes regulated by Pint and bound by Suz12 [31].

Additional file 8: Table S7 Information on the human samples used in this study.

\section{Abbreviations}

5-FU: fluorouracil; ASO: Anti-sense oligonucleotide; BrdU: bromodeoxyuridine; ChIP: chromatin immunoprecipitation; ChIP-seq: ChIP sequencing; Ezh2: Enhancer of zeste homolog 2; FACS: fluorescence-activated cell sorting; FISH: Fluorescence in situ hybridization; KEGG: Kyoto Encyclopedia of Genes and Genomes; lincRNA: Long intergenic non-coding RNA; Mapk: mitogenactivated protein kinase; MEF: mouse embryonic fibroblast; mESC: mouse embryonic stem cell; ORF: Open reading frame; PINT: p53 induced noncoding transcript; PRC2: Polycomb repressive complex 2; RT-GPCR:

Quantitative real time PCR; RACE: rapid amplification of CDNA ends; RIP-seq: RNA immunoprecipitation sequencing; RNAi: RNA interference; shRNA: short hairpin RNA; siRNA: small interfering RNA; Suz12: Suppressor of zeste 12; Tgf: transforming growth factor; Wdr5: WD repeat-containing protein 5.

\section{Competing interests}

The authors declare that they have no competing interests.

\section{Authors' contributions}

$\mathrm{MH}$ and $\mathrm{OMB}$ designed the research; OMB, FM, YS, and JG performed the research; AA, VS, and J R performed data analysis; IM, AN, MM, and JGF contributed to the collection and analysis of data from human samples; SG contributed to design, testing and production of reagents; and $\mathrm{MH}$ and OMB wrote the paper.

\section{Acknowledgements}

We thank Tomás Aragón for critical reading of the manuscript. This research was supported by a European Research Council Starting Grant (number 281877) and Spanish Ministry of Science Grants (numbers BFU2011-23485 and SRYC11001008347XV0).

\section{Authors' details}

'Center for Applied Medical Research, University of Navarra, 55 Pio XII Avenue., 31008 Pamplona, Spain. ${ }^{2}$ Department of Antisense Drug Discovery, Isis Pharmaceuticals, 2855 Gazelle Court., Carlsbad, CA 92008, USA.

${ }^{3}$ Department of Medical Oncology, Hospital Municipal de Badalona, 9-13 Via Augusta Street, 08911 Badalona, Spain. ${ }^{4}$ Molecular Oncology and

Embryology Laboratory, Human Anatomy Unit, University of Barcelona Medical School, IDIBAPS, 143 Casanova Street, 080360 Barcelona, Spain. ${ }^{5}$ Department of Oncology, Translational Oncology Division, Health Research Institute Fundación Jiménez Díaz University Hospital, Autonomous University of Madrid, 2 Reyes Catolicos Avenue, 28040 Madrid, Spain. ${ }^{6}$ Stem Cell and Regenerative Biology, Harvard University, 7 Divinity Avenue, Cambridge, MA 02138, USA.

Received: 24 April 2013 Revised: 11 July 2013

Accepted: 26 September 2013 Published: 26 September 2013

\section{References}

1. Beckerman R, Prives C: Transcriptional regulation by p53. Cold Spring Harb Perspect Biol 2010, 2(8):a000935.

2. He L, He X, Lim LP, de Stanchina E, Xuan Z, Liang Y, Xue W, Zender L, Magnus J, Ridzon D, Jackson AL, Linsley PS, Chen C, Lowe SW, Cleary MA, 
Hannon GJ: A microRNA component of the p53 tumour suppressor network. Nature 2007, 447:1130-1134.

3. Huarte M, Guttman M, Feldser D, Garber M, Koziol MJ, Kenzelmann-Broz D, Khalil AM, Zuk O, Amit I, Rabani M, Attardi LD, Regev A, Lander ES, Jacks T, Rinn J: A large intergenic noncoding RNA induced by p53 mediates global gene repression in the p53 response. Cell 2010, 142:409-419.

4. Hung T, Wang Y, Lin MF, Koegel AK, Kotake Y, Grant GD, Horlings HM, Shah N, Umbricht C, Wang P, Wang Y, Kong B, Langerød A, BørresenDale AL, Kim SK, van de Vijver M, Sukumar S, Whitfield ML, Kellis M, Xiong Y, Wong DJ, Chang HY: Extensive and coordinated transcription of noncoding RNAs within cell-cycle promoters. Nat Genet 2011, 43:621-629.

5. Mercer TR, Dinger ME, Mattick JS: Long non-coding RNAs: insights into functions. Nat Rev Genet 2009, 10:155-159.

6. Rinn $\mathrm{J}$, Chang HY: Genome regulation by long noncoding RNAs. Annu Rev Biochem 2012, 81:145-166.

7. Wang KC, Chang HY: Molecular mechanisms of long noncoding RNAs. Mol Cell 2011, 43:904-914.

8. Guttman M, Rinn JL: Modular regulatory principles of large non-coding RNAs. Nature 2012, 482:339-346.

9. Mattick JS, Amaral PP, Dinger ME, Mercer TR, Mehler MF: RNA regulation of epigenetic processes. Bioessays 2009, 31:51-59.

10. Khalil AM, Guttman M, Huarte M, Garber M, Raj A, Rivea Morales D, Thomas K, Presser A, Bernstein BE, van Oudenaarden A, Regev A, Lander ES, Rinn JL: Many human large intergenic noncoding RNAs associate with chromatin-modifying complexes and affect gene expression. Proc Natl Acad Sci USA 2009, 106:11667-11672.

11. Koziol MJ, Rinn JL: RNA traffic control of chromatin complexes. Curr Opin Genet Dev 2010, 20:142-148.

12. Sparmann A, van Lohuizen M: Polycomb silencers control cell fate, development and cancer. Nat Rev Cancer 2006, 6:846-856.

13. Kuzmichev A, Margueron R, Vaquero A, Preissner TS, Scher M, Kirmizis A, Ouyang X, Brockdorff N, Abate-Shen C, Farnham P, Reinberg D: Composition and histone substrates of polycomb repressive group complexes change during cellular differentiation. Proc Natl Acad Sci USA 2005, 102:1859-1864.

14. van Kemenade FJ, Raaphorst FM, Blokzijl T, Fieret E, Hamer KM, Satijn DP, Otte AP, Meijer CJ: Roles of the EZH2 histone methyltransferase in coexpression of BMI-1 and EZH2 polycomb-group proteins is associated with cycling cells and degree of malignancy in B-cell non-Hodgkin lymphoma. Blood 2001, 97:3896-3901.

15. Simon JA, Lange CA: cancer epigenetics. Mutat Res 2008, 647:21-29.

16. Ting AH, McGarvey KM, Baylin SB: The cancer epigenome-components and functional correlates. Genes Dev 2006, 20:3215-3231.

17. Wapinski O, Chang HY: Long noncoding RNAs and human disease. Trends Cell Biol 2011, 21:354-361

18. Esteller M: Non-coding RNAs in human disease. Nat Rev Genet 2011, 12:861-874.

19. Garber M, Guttman M, Clamp M, Zody MC, Friedman N, Xie X: Identifying novel constrained elements by exploiting biased substitution patterns. Bioinformatics 2009, 25:154-62.

20. Li M, He Y, Dubois W, Wu X, Shi J, Huang J: Distinct regulatory mechanisms and functions for p53-activated and p53-repressed DNA damage response genes in embryonic stem cells. Mol Cell 2012, 46:30-42.

21. Kenzelmann Broz D, Spano Mello S, Bieging KT, Jiang D, Dusek RL, Brady CA, Sidow A, Attardi LD: Global genomic profiling reveals an extensive p53-regulated autophagy program contributing to key p53 responses. Genes Dev 2013, 27:1016-1031.

22. Clamp M, Fry B, Kamal M, Xie X, Cuff J, Lin MF, Kellis M, Lindblad-Toh K, Lander ES: Distinguishing protein-coding and noncoding genes in the human genome. Proc Natl Acad Sci USA 2007, 104:19428-19433.

23. Ventura A, Kirsch DG, McLaughlin ME, Tuveson DA, Grimm J, Lintault L, Newman J, Reczek EE, Weissleder R, Jacks T: Restoration of p53 function leads to tumour regression in vivo. Nature 2007, 445:661-665.

24. Bennett CF, Swayze EE: RNA targeting therapeutics: molecular mechanisms of antisense oligonucleotides as a therapeutic platform. Annu Rev Pharmacol Toxicol 2010, 50:259-293.

25. Vickers TA, Koo S, Bennett CF, Crooke ST, Dean NM, Baker BF: Efficient reduction of target RNAs by small interfering RNA and RNase $\mathrm{H}$ dependent antisense agents. A comparative analysis. J Biol Chem 2003, 278:7108-7118.
26. Zhao J, Ohsumi TK, Kung JT, Ogawa Y, Grau DJ, Sarma K, Song JJ, Kingston RE, Borowsky M, Lee JT: Genome-wide identification of polycomb-associated RNAs by RIP-seq. Mol Cell 2010, 40:939-953.

27. Guil S, Soler M, Portela A, Carrere J, Fonalleras E, Gomez A, Villanueva A, Esteller M: Intronic RNAs mediate EZH2 regulation of epigenetic targets. Nat Struct Mol Biol 2012, 19:664-670.

28. Rinn JL, Kertesz M, Wang JK, Squazzo SL, Xu X, Brugmann SA, Goodnough LH, Helms JA, Farnham PJ, Segal E, Chang HY: Functional demarcation of active and silent chromatin domains in human HOX loci by noncoding RNAs. Cell 2007, 129:1311-1323.

29. Wang KC, Yang YW, Liu B, Sanyal A, Corces-Zimmerman R, Chen Y, Lajoie BR, Protacio A, Flynn RA, Gupta RA, Wysocka J, Lei M, Dekker J, Helms JA, Chang HY: A long noncoding RNA maintains active chromatin to coordinate homeotic gene expression. Nature 2011, 472:120-124.

30. Nagano T, Mitchell JA, Sanz LA, Pauler FM, Ferguson-Smith AC, Feil R, Fraser P: The Air noncoding RNA epigenetically silences transcription by targeting G9a to chromatin. Science 2008, 322:1717-1720.

31. Ku M, Koche RP, Rheinbay E, Mendenhall EM, Endoh M, Mikkelsen TS, Presser A, Nusbaum C, Xie X, Chi AS, Adli M, Kasif S, Ptaszek LM, Cowan CA, Lander ES, Koseki H, Bernstein BE: Genomewide analysis of PRC1 and PRC2 occupancy identifies two classes of bivalent domains. PLoS Genet 2008, 4:e1000242.

32. Mikkelsen TS, Ku M, Jaffe DB, Issac B, Lieberman E, Giannoukos G, Alvarez P, Brockman W, Kim TK, Koche RP, Lee W, Mendenhall E, O'Donovan A, Presser A, Russ C, Xie X, Meissner A, Wernig M, Jaenisch R, Nusbaum C, Lander ES, Bernstein BE: Genome-wide maps of chromatin state in pluripotent and lineage-committed cells. Nature 2007, 448:553-560.

33. Bunz F, Dutriaux A, Lengauer C, Waldman T, Zhou S, Brown JP, Sedivy JM, Kinzler KW, Vogelstein B: Requirement for $\mathrm{p} 53$ and p21 to sustain G2 arrest after DNA damage. Science 1998, 282:1497-1501.

34. Perez-Llamas C, Lopez-Bigas N: Gitools: analysis and visualisation of genomic data using interactive heat-maps. PLoS One 2011, 6:e19541.

35. Guttman M, Amit I, Garber M, French C, Lin MF, Feldser D, Huarte M, Zuk O, Carey BW, Cassady JP, Cabili MN, Jaenisch R, Mikkelsen TS, Jacks T, Hacohen N, Bernstein BE, Kellis M, Regev A, Rinn JL, Lander ES: Chromatin signature reveals over a thousand highly conserved large non-coding RNAs in mammals. Nature 2009, 458:223-227.

36. Cabili MN, Trapnell C, Goff L, Koziol M, Tazon-Vega B, Regev A, Rinn JL: Integrative annotation of human large intergenic noncoding RNAs reveals global properties and specific subclasses. Genes Dev 2011, 25:1915-1927.

37. Zhang A, Zhou N, Huang J, Liu Q, Fukuda K, Ma D, Lu Z, Bai C, Watabe K, Mo YY: The human long non-coding RNA-RoR is a p53 repressor in response to DNA damage. Cell Res 2012, 23:340-350.

38. Liu Q, Huang J, Zhou N, Zhang Z, Zhang A, Lu Z, Wu F, Mo YY: LncRNA loc285194 is a p53-regulated tumor suppressor. Nucleic Acids Res 2013

39. Martianov I, Ramadass A, Serra Barros A, Chow N, Akoulitchev A: Repression of the human dihydrofolate reductase gene by a non-coding interfering transcript. Nature 2007, 445:666-670.

40. Schmitz KM, Mayer C, Postepska A, Grummt I: Interaction of noncoding RNA with the rDNA promoter mediates recruitment of DNMT3b and silencing of rRNA genes. Genes Dev 2010, 24:2264-2269.

41. Tsai MC, Manor O, Wan Y, Mosammaparast N, Wang JK, Lan F, Shi Y, Segal E, Chang HY: Long noncoding RNA as modular scaffold of histone modification complexes. Science 2010, 329:689-693.

42. Toledo F, Wahl GM: Regulating the p53 pathway: in vitro hypotheses, in vivo veritas. Nat Rev Cancer 2006, 6:909-923.

43. Feldser DM, Kostova KK, Winslow MM, Taylor SE, Cashman C, Whittaker CA, Sanchez-Rivera FJ, Resnick R, Bronson R, Hemann MT, Jacks T: Stagespecific sensitivity to $\mathrm{p} 53$ restoration during lung cancer progression. Nature 2010, 468:572-575.

44. Huarte M, Rinn JL: Large non-coding RNAs: missing links in cancer?. Hum Mol Genet 2010, 19:R152-161.

45. Moffat J, Grueneberg DA, Yang X, Kim SY, Kloepfer AM, Hinkle G, Piqani B, Eisenhaure TM, Luo B, Grenier JK, Carpenter AE, Foo SY, Stewart SA, Stockwell BR, Hacohen N, Hahn WC, Lander ES, Sabatini DM, Root DE: A lentiviral RNAi library for human and mouse genes applied to an arrayed viral high-content screen. Cell 2006, 124:1283-1298.

doi:10.1186/gb-2013-14-9-r104

Cite this article as: Marín-Béjar et al:: Pint lincRNA connects the p53 pathway with epigenetic silencing by the Polycomb repressive complex 2. Genome Biology 2013 14:R104. 